\title{
Wnt5a-induced M2 polarization of tumor-associated macrophages via IL- 10 promotes colorectal cancer progression
}

\author{
Qing Liu ( $\square$ liuqing136@whu.edu.cn ) \\ Wuhan University \\ Chaogang Yang \\ Wuhan University Zhongnan Hospital \\ Shuyi Wang \\ Wuhan University Zhongnan Hospital \\ Dongdong Shi \\ Wuhan University Zhongnan Hospital \\ Chen Wei \\ Wuhan University Zhongnan Hospital \\ Jialin Song \\ Wuhan University Zhongnan Hospital \\ Xiaobin Lin \\ Wuhan University Zhongnan Hospital \\ Rongzhang Dou \\ Wuhan University Zhongnan Hospital \\ Jian Bai \\ Wuhan University Zhongnan Hospital \\ Zhenxian Xiang \\ Wuhan University Zhongnan Hospital \\ Sihao Huang \\ Wuhan University Zhongnan Hospital \\ Keshu Liu \\ Wuhan University Zhongnan Hospital \\ Bin Xiong \\ Wuhan University Zhongnan Hospital
}

\section{Research}

Keywords: Tumor-associated macrophages, Wnt5a, IL-10, M2 polarization, colorectal cancer

Posted Date: March 17th, 2020

DOI: https://doi.org/10.21203/rs.2.18621/v2

License: (c) (7) This work is licensed under a Creative Commons Attribution 4.0 International License. Read Full License

Version of Record: A version of this preprint was published at Cell Communication and Signaling on March 30th, 2020. See the published version at https://doi.org/10.1186/s12964-020-00557-2. 


\section{Abstract}

Background: Tumor-associated macrophages (TAMs) in the tumor microenvironment influence tumor initiation, invasion and metastasis. Several studies have shown that Wnt5a is mainly expressed in the tumor stroma, especially in TAMs. However, whether Wnt5a regulates the polarization and biological function of TAMs in colorectal cancer (CRC) is incompletely understood.

Methods: Immunofluorescence staining was performed to detect CD68 and Wnt5a expression in colorectal tissues from patients (63 CRC specimens VS 20 normal tissues). RT-qPCR, flow cytometry, ELISA and inhibitors were carried out to explore the role of Wnt5a in the polarization of TAMs. Clone formation and transwell assays were performed to determine the effects of Wnt5a-treated macrophages on tumor proliferation, migration and invasion in vitro. Finally, a xenograft model was applied to confirm the effects of Wnt5a + TAMs on CRC tumorigenesis.

Results: We found that high Wnt5a + CD68 + /CD68 + TAMs ratio was significantly associated with poor prognosis in CRC patients and Wnt5a + TAM was an M2-like TAM subtype. Subsequently, we found that Wnt5a induced macrophages to secrete IL-10, which then acted as an autocrine cytokine to induce M2 polarization of these macrophages. IL-10 neutralizing antibody completely reversed the pro-M2 effect of Wnt5a. Mechanistically, the CaKMII-ERK1/2-STAT3 pathway was required for Wnt5a-mediated IL-10 expression in macrophages. Furthermore, Wnt5ainduced M2 macrophages promoted CRC cells proliferation, migration and invasion; knockdown of Wnt5a in TAMs significantly impaired the protumor functions of TAMs.

Conclusions: Our data indicate that Wnt5a could induce M2 polarization of TAMs by regulating CaKMII-ERK1/2-STAT3 pathway-mediated IL-10 secretion, ultimately promoting tumor growth and metastasis of CRC.

\section{Background}

Metastasis is a major cause of cancer-related deaths(1). Tumor microenvironment (TME) is closely correlated with tumor metastasis, growth and occurrence(2, 3). TME is mainly composed of cancer cells, cancer-associated fibroblasts, immune cells and the non-cellular components(4). As the most abundant immune cells in TME, tumor-associated macrophages (TAMs) can secrete various mediators such as cytokines or chemokines to suppress anti-tumor immune response, stimulate angiogenesis and finally enhance cancer cells proliferation, invasion, intravasation and dissemination for metastasis(5-7). As TAMs exert an important role in cancer metastasis, our group studied the interaction between TAMs and cancer cells. We demonstrated that TAMs, especially M2 subtype, induced epithelial-mesenchymal transition (EMT) of cancer cells to promote circulating tumor cell-mediated colorectal cancer (CRC) metastasis(8). Meanwhile, we also found that EMT-programmed cancer cells could remodel the tumor microenvironment and activate TAMs to M2 phenotype(9), thereby facilitating tumor progression and metastasis(10, 11). Therefore, M2like TAMs might be critical players in the crosstalk between cancer cells and their microenvironment(10, 12). Although the cancer-promoting functions of M2-like TAMs are well defined, the potential mechanisms underlying M2-like TAMs polarization still need further exploration.

M2 polarization of TAMs is a multi-factor, multi-step and complex pathological process(13). Recent studies have shown that Wnt signaling plays a major role in M2-like TAMs polarization $(14,15)$. Wnt signaling pathway is generally considered to be divided into two types-canonical Wnt/ $\beta$ catenin signaling and noncanonical Wnt signaling, which is mainly activated by 19 Wnt proteins identified in mammals(16).Wnt5a, a member of Wnt family, binds to the frizzled receptor and LRP5/6 co-receptors to regulate multiple signaling pathways(17), thereby altering TME by inducing adipocytes to dedifferentiate into fibroblast-like cells or mesenchymal cells(18). Furthermore, Wnt5a was considered to participate in the differentiation of immune cell, including dendritic cells, B cells, as well as macrophages(19-21). Previously, Wnt5a was mainly located in the TAMs of tumor stroma in breast cancer or basal cell carcinoma(22, 23); Smith and colleagues consistently found that Wnt5a was mostly expressed in the tumor stroma of CRC, especially in TAMs(24). These findings indicated that Wnt5a could be involved in the regulation of tumor immune microenvironment. Moreover, Bergenfelz et al. observed that Wnt5a was mainly located in $\mathrm{CD}_{163^{+}}$TAMs in breast cancer and could induce a tolerogenic phenotype of macrophages(25). Based on the above studies and our previous results $(8,9)$, we speculated that Wnt5a might induce $\mathrm{M} 2$ polarization of TAMs to promote tumor development in CRC.

In the present study, we attempted to investigate the role and underlying mechanism of Wnt5a in the reprogramming of TAMs by a serial of clinical samples analyses, in vitro and in vivo experiments. Our results showed that high Wnt5 ${ }^{+} \mathrm{CD} 68^{+} / \mathrm{CD} 68^{+}$TAMs ratio was significantly associated with poor prognosis in CRC patients. Wnt5a induced M2 polarization of TAMs by regulating CaKMII-ERK1/2-STAT3 pathway-mediated IL-10 secretion, thereby enhancing tumor growth, invasion and metastasis of CRC. These findings unveil a new insight into understanding the indirect role of Wnt5a in CRC progression.

\section{Materials And Methods}

\section{Patients and specimens}

A total of $63 \mathrm{CRC}$ samples and 20 normal colorectal tissues were collected from patients who were diagnosed as primary CRC by histopathology and underwent curative resection at the Zhongnan Hospital of Wuhan University (Wuhan, China) between January 2013 and April 2015 . No patients had received adjuvant preoperative chemotherapy or radiotherapy. Moreover, all enrolled patients had available clinicopathological 
characteristics information and follow-up data. Formalin-fixed, paraffin-embedded tumor specimens were prepared after surgical resection. Informed consents were obtained from these patients. The study protocol was approved by the research ethics committee of Zhongnan Hospital of Wuhan University.

\section{Immunofluorescence staining}

The Paraffin-embedded specimens were sectioned at $3 \mu \mathrm{m}$ thickness. After dewaxing, antigen retrieval was made in the microwave of citrate buffer. The slices were then blocked with $3 \% \mathrm{BSA}$ and stained overnight at $4^{\circ} \mathrm{C}$ with the primary antibodies against human Wnt5a (1:100, $\left.\mathrm{Abcam}\right), \mathrm{CD} 68$ (1:100, Abcam), HLA-DR (1:100, Abcam), CD163 (1: 100, Abcam), IL-10 (1:100, Abcam). Subsequently, specimen sections were incubated with fluorochrome-conjugated secondary antibodies. Nucleus staining was performed using Diaminophenylindole (DAPI).

Before immunofluorescence staining, all samples were stained with hematoxylin and eosin (H\&E) to ensure that all specimens contained representative CRC tissue. For each section, at least 6 fields of view (at 200x magnification) were used to calculate positive cells stained with indicated antibody. A quantitative analysis was performed to determine the numbers of infiltrating $\mathrm{CD} 68^{+}$and $\mathrm{Wnt}^{2} \mathrm{a}^{+} \mathrm{CD} 68^{+}$cells. All of the abovementioned cells were counted manually. Only cells with macrophage-like morphology were included. Cell counting was carried out in a blinded manner by two research fellows familiar with histomorphology and immunofluorescence staining. If there was a disagreement, the analyses would be discussed with the authors.

\section{Cell culture and reagents}

Human CRC cell lines (SW480, HT29, HCT116, DLD-1) were obtained from the cell bank of Chinese Academy of Sciences in Shanghai. Human monocyte cell line THP-1 was purchased from ATCC. Cells were cultured in RPMI 1640 medium (Hyclone) with 10\% heat-inactivated fetal bovine serum (Wisent) and incubated in a humidified atmosphere with $5 \% \mathrm{CO} 2$ at a constant temperature of $37^{\circ} \mathrm{C}$. Human recombinant Wnt5a and IL- 10 protein were purchased from R\&D Systems and were used at the final concentration of 500 $\mathrm{ng} / \mathrm{ml}$ and $50 \mathrm{ng} / \mathrm{ml}$ respectively. Box 5 (Wnt5a inhibitor, Merck, USA), U0126 (p-ERK inhibitor, Merck, USA), S3I-201 (p-STAT3 inhibitor, Merck, USA), CK59 (p-CaMKII inhibitor, Merck, USA) were used at $100 \mu \mathrm{M}, 20 \mathrm{ng} / \mathrm{ml}, 100 \mu \mathrm{M}, 50 \mu \mathrm{M}$, respectively. Human neutralizing IL-10 antibody and isotype IgG were purchased from Biolegend, USA.

\section{Macrophage generation and differentiation}

THP-1 cells were first treated with $100 \mathrm{ng} / \mathrm{ml}$ PMA (Sigma-Aldrich, USA) for $24 \mathrm{~h}$ to generate THP-1 macrophages (M0 macrophages), followed by induction with IL-4 (50 ng/ml, R\&D Systems)/IL-13 (20 ng/ml, R\&D Systems) or LPS (100 ng/ml, Absin)/ IFN-y (20 ng/ml, Absin) for $48 \mathrm{~h}$ to differentiate into M2 or M1 macrophages. To mimic TAMs formation, M0 macrophages were co-cultured with colorectal cancer cells (HCT116 or DLD-1) in a 6-well transwell co-cultivation system $(0.4 \mu \mathrm{m}$ pore size, Corning, USA). After $48 \mathrm{~h}$, the co-cultured macrophages were collected to obtain TAMs.

\section{ELISA}

The level of Wnt5a and IL-10 was estimated in different culture media by an ELISA kit (R\&D Systems) according to the manufacturer's instructions. The presented value represented the average of 3 independent experiments.

\section{Flow cytometry}

Macrophages were harvested from 6-well plates, washed twice with PBS, made into single-cell suspensions, and then incubated with antibodies (FITC Mouse anti-Human CD68, PE Mouse Anti-human CD163, APC Mouse anti-Human HLA-DR, all from BD Biosciences, USA) for 60 min at $4{ }^{\circ} \mathrm{C}$. The stained cells were then washed twice and resuspended in the 200 $\mu$ l flow buffer for analysis on a FACS Calibur flow cytometer (BD Biosciences, USA) using FlowJo software (FlowJo, USA).

\section{Reverse transcription-quantitative PCR (RT-qPCR)}


Total RNAs were extracted using the Trizol Reagent (Invitrogen, USA) according to the manufacturer's instructions. Then 2ug RNA was reverse transcribed to synthesize cDNA using the Primescript ${ }^{T M}$ RT reagent Kit (Vazyme, Nanjing, China), followed by RT-qPCR using the SYBR-Green PCR Master Mix (Vazyme, Nanjing, China), which was performed on the BIO-RAD CFX96 Real-time PCR machine. The relative expression level of mRNA was computed by using $2^{-\Delta \Delta C t}$ method. The sequences of all primers are listed in Table $S 2$.

\section{Western blot}

Total cellular proteins were extracted using RIPA lysis reagent (Aspen) containing a protease inhibitor cocktail (Aspen) with or without a phosphatase inhibitor (Bio-swamp). The protein concentration was measured by BCA protein assay reagent kit (Aspen) and mixed with $4 \times$ SDS loading buffer (Bio-swamp) for denaturation in a $100^{\circ} \mathrm{C}$ boiling water bath for $8 \mathrm{~min}$. The proteins were then separated on $10 \%$ SDS-PAGE gels and transferred to PVDF membranes (Merck). The PVDF membranes were blocked with 5\% skim milk for 90 min, washed three times, and then incubated with primary antibody at $4^{\circ} \mathrm{C}$ overnight. Thereafter, the PVDF membranes were washed three times for 30 min and incubated with HRPconjugated secondary antibodies for $90 \mathrm{~min}$ at room temperature. Finally, the membranes were again washed five times for $30 \mathrm{~min}$, exposed to ECL developer (Aspen) and analyzed by Bio-Rad Image Lab software. The following primary antibodies used in this study were listed: Wnt5a (1:1000, Abcam), ERK1/2 (1:1000, Protein Tech), p-ERK1/2 ( 1:1000, CST), STAT3 (1:1000, Protein Tech), p-STAT3 (1:1000, CST), NF-kB p65 (1:1000, Protein Tech), p-NF-kB p65 (1:1000, CST), CaMKII (1:1000, Protein Tech), p-CaMKII ( 1:1000, CST), GAPDH (1:5000, Protein Tech), a-Tubulin (1:5000, Protein Tech).

\section{Cell transfection}

Lentiviral-mediated shRNA interference was performed as described previously(26). Wnt5a expression was knocked down in SW480 or TAMs by transduction of a lentiviral vector expressing a short hairpin RNA-Wnt5a shRNA (sh-Wnt5a) or negative control shRNA (sh-NC) (Genechem, China). Transfections were performed using X-treme GENE HP reagent (Roche, USA) according to the manufacturer's instructions.

\section{CCK-8 and clone formation assay}

Cell viability was estimated using a CCK-8 reagent kit (Vazyme, Nanjing, China). 5000 CRC cells were planted in 96-well plates and cultured for indicated time, followed by addition of CCK-8 solution. Then, the spectral absorbance in each well was quantified at $450 \mathrm{~nm}$ using a microplate reader (Molecular Devices, USA). For colony formation detection, co-cultured HCT116 or DLD-1 cells were washed, digested, counted and seeded with 500 cells per well in a 6-well plate. After 2 weeks, colonies were fixed with $4 \%$ paraformaldehyde and then stained with $0.5 \%$ crystal violet (Sigma). Each clone that had more than 50 cells was counted. All experiments were performed in triplicate.

\section{Transwell migration and invasion assay}

The experiments were performed using a 24-well transwell system ( $8 \mu \mathrm{m}$ pore size; Corning, USA). For the migration assay, co-cultured $5 \times 10^{4}$ HCT116 or DLD-1 cells were suspended in $600 \mu$ RPMI 1640 containing 1\% FBS and seeded into the upper chamber, while $700 \mu$ l RPMI 1640 containing $10 \%$ FBS was added to the lower chamber. After incubation for $48 \mathrm{~h}$, the chamber was fixed with $4 \%$ paraformaldehyde and then stained with $0.5 \%$ crystal violet. Cells in the upper chamber were removed with a cotton swab. Cells in 4 randomly microscopic fields (at $\times 100$ magnification) were counted and photographed. For the invasion assay, the upper chamber was coated with Matrigel (BD Biosciences, USA) before inoculation. The remaining steps were the same as the migration assay. Each experiment was performed in triplicates.

\section{In vivo experiments}

Twenty-four BALB/c female nude mice (6-8 weeks old and 20-25 g) were purchased from the Hubei Research Center of Laboratory Animals (Wuhan, China). All animal experiments were conducted strictly in compliance with the guidelines of the Institutional Animal Care and Use Committee of the Zhongnan Hospital of Wuhan University. Nude mice were randomly divided into 4 groups ( $n=6$ per group). HCT116 cells alone (5 $\left.\times 10^{5}\right)$, TAMs alone $\left(1 \times 10^{5}\right)$, HCT116 cells $\left(5 \times 10^{5}\right)+$ sh-NC TAMs $\left(1 \times 10^{5}\right)$, HCT116 cells $\left(5 \times 10^{5}\right)+$ sh-Wnt5a TAMs $\left(1 \times 10^{5}\right)$ were separately mixed, resuspended with 200ul PBS, and then subcutaneously injected into the right flank of mice. After 10 days, the size of the tumors was measured with digital vernier calipers once a week. The volume of the tumors was calculated according to the formula: volume $=($ length $x$ width $\left.^{2}\right) / 2$. After 45 days of cells inoculation, $1 \mathrm{ml}$ of blood per mouse was harvested by cardiac puncture into EDTA-containing tubes, and then 
nude mice were sacrificed. Tumor tissues were collected and measured for weight and volume, followed for further analysis by RT-qPCR assay or IHC staining. In addition, liver and lung tissues of mice were collected to further evaluate the metastatic burden by H\&E staining.

\section{Immunohistochemistry}

The sample sections were subjected to dewaxing, antigen retrieval, incubation with $3 \%$ hydrogen peroxide solution and $3 \%$ BSA blocking. Slides were subsequently incubated with primary antibodies against human Wnt5a (1:100, Abcam), CD163 (1:100, Abcam), IL-10 (1:100, Abcam), $\mathrm{Ki67}(1: 500, \mathrm{Abcam})$ at $4^{\circ} \mathrm{C}$ overnight, followed by incubation with HRP-labeled secondary antibodies. Immunoreactions were visualized using enzymatic Avidin-Biotin Complex ( $A B C$ )-diaminobenzidine (DAB).

\section{CTC enrichment}

Circulating tumor cells (CTCs) were captured, isolated, and identified by a novel CTCBIOPSY method as reported by our previous study(8, 9, 27). Briefly, $1 \mathrm{ml}$ of blood of mice was first diluted with $4 \mathrm{ml}$ of $0.9 \%$ physiological saline and then totally transferred to CTCBIOPSY tubes with an $8-\mu \mathrm{m}$ diameter aperture membrane. The diluted blood samples were filtered by positive pressure through the membrane, where CTCs were captured. CTCs on the membrane were stained with the primary antibodies (CK, CD45, Hoechst, all from Abcam) and then counted under a microscope (BX51-Olympus, Japan).

\section{Statistics analysis}

All results were presented as means \pm SEM from at least three independent experiments. Statistical analyses were performed using SPSS statistical software (version 19.0, IBM, USA). Means of continuous outcome variables were appropriately tested with one-way analyses of variance or two-tailed Student's t-test. The clinical feature correlation analysis was performed using chi-squared test. Kaplan-Meier curves were applied to survival analysis, and log-rank test was used to assess differences in different subgroups of patients. Univariate and multivariate Cox-regression analyses were performed to determine independent prognostic factors. Differences were considered statistically significant when $p$ values $<0.05$.

\section{Results}

\section{The ratio of Wnt5a ${ }^{+} \mathrm{CD} 68^{+} / \mathrm{CD} 68^{+}$TAMs is correlated with poor prognosis in CRC patients}

In order to determine the relationship between Wnt5a and TAMs, we first detected the expression of Wnt5a and TAM marker (CD68) in 20 normal colorectal tissues and 63 CRC specimens. Immunofluorescence analysis revealed that Wnt5a expression was markedly elevated in CRC compared with normal tissue (Fig.1A). Intriguingly, we found that Wnt5a was mainly expressed in the tumor stroma, especially in TAMs, with no or scarce expression in tumor nest (Fig.1A, Figure S1A). Moreover, we firstly observed that the number of Wnt5a ${ }^{+}$TAMs was significantly increased in CRC samples compared to normal tissues (Figure S1B). High Wnt5a+ TAMs expression was significantly correlated with LVI and TNM stage (Table S1). Further survival analysis showed that high Wnt5a+ TAMs expression was significantly associated with poor RFS and OS (Figure S1 C and D), while $\mathrm{CD}^{\circ} 8^{+}$TAMs expression was insignificantly correlated with the prognosis of CRC patients (Figure S1E and F). We then estimated the significance of Wnt5a ${ }^{+} \mathrm{CD} 68^{+} / \mathrm{CD} 68^{+}$TAMs ratio. We categorized all $\mathrm{CRC}$ patients into high and low $\mathrm{Wnt} \mathrm{a}^{+} \mathrm{CD} 68^{+} / \mathrm{CD} 68^{+}$ratio groups using the median ratio value as a cutoff threshold. Similar to Wnt5a ${ }^{+} \mathrm{TAMs}$, Wnt5a ${ }^{+} \mathrm{CD} 68^{+} / \mathrm{CD} 68^{+} \mathrm{TAMs}$ ratio was dramatically higher in $\mathrm{CRC}$ specimens than that in normal tissues (Fig.1B). High Wnt5a ${ }^{+} \mathrm{CD} 68^{+} / \mathrm{CD} 68^{+}$ratio was significantly correlated with LVI, TI, LNM and TNM stage (Table 1 ). Further survival analysis showed that a higher ratio of Wnt5a ${ }^{+} \mathrm{CD} 68^{+} / \mathrm{CD} 68^{+} \mathrm{TAMs}$ was significantly associated with worse RFS and OS (Fig. $1 \mathrm{C}$ and D). Univariate and multivariate analyses revealed that only Wnt5a ${ }^{+} \mathrm{CD} 68^{+} / \mathrm{CD} 68^{+}$TAMs ratio in the above-mentioned indicators was an independent prognostic factor associated with poor RFS and OS (Table 2). These results indicate that Wnt5a+ TAM is a poor prognostic indicator, and the ratio of Wnt5a ${ }^{+} \mathrm{CD} 68^{+} / \mathrm{CD} 68^{+}$TAMs can serve as a marker for evaluating prognosis. Furthermore, a higher Wnt5a ${ }^{+} \mathrm{CD} 68^{+} / \mathrm{CD} 68^{+}$ratio was observed at the tumor invasive front (Fig. 1E and F), where there exists M2-like TAMs infiltration(8, 12). So, we speculated that Wnt5a+ TAM might be an M2-like TAM subtype. Further immunofluorescence analysis showed that Wnt5a was mainly co-expressed with CD163 (M2 marker) but not with HLA-DR (M1 marker) (Fig. 1G).

\section{Wnt5a is mainly expressed in M2-like TAMs}


To validate the above clinical results, we applied an in vitro model of tumor-associated macrophages according to previous reports(28). As shown in the flowchart (Fig. 2A), after treated with PMA for 24h, human THP-1 monocytes were differentiated into M0 macrophages and then co-cultured with CRC cells (HCT116 or DLD-1) for 48h to generate TAMs. TAMs exhibited higher levels of M2 markers CD163, CD206, and lower levels of M1 marker HLA-DR (Fig. 2B). Flow cytometry analysis showed that the proportion of CD163 positive cells in TAMs was around 33.6\%, and 43.7\% in IL4/IL-13-induced M2 macrophages (Fig. 2C). Additionally, TAMs also expressed higher levels of M2 markers IL-10, TGF- $\beta$, CCL17, CCL18 and CCL22 and lower level of M1 marker IL-12 (Fig. 2B). These results suggest that TAM produced by the in vitro model is a kind of macrophage based on M2 phenotype.

We then investigated Wnt5a expression in different phenotypes of macrophages. As shown in Fig. 2D and Figure S2A, Wnt5a was apparently overexpressed in TAMs and M2 macrophages, while scarcely expressed in M0 and M1 macrophages. Wnt5a expression in CRC cell lines was also rare or scarce (Figure S2B). Further ELISA analysis showed that the secretion of Wn5a in TAMs was much more than that in M0 macrophages or CRC cells (HCT116 or DLD-1) (Fig. 2E). In addition, cellular immunofluorescence confirmed that Wnt5a was mainly expressed in CD163 ${ }^{+}$TAMs (Fig. 2F). Together, our findings reveal that Wnt5a is primarily located in M2-like TAMs.

\section{Wnt5a induces M2 macrophage polarization via IL-10}

Based on the above results and previous research, we assumed that Wnt5a was an important factor affecting M2 polarization. To assess the role of Wnt5a in macrophage polarization, we first treated M0 macrophages with $500 \mathrm{ng} / \mathrm{ml}$ Wnt5a for 3 days. RT-qPCR analysis showed that the expression of CD163 and IL-10 were significantly up-regulated in Wnt5a-stimulated M0 macrophages compared to untreated M0 macrophages (Fig. 3A). Subsequently, we tested the percentage of $\mathrm{CD}_{163^{+}}$cells by flow cytometry in M0 macrophages treated with Wnt5a. We found nearly $9.8 \%$ $\mathrm{CD}_{163^{+}}$macrophages in Wnt5a-stimulated M0 macrophages, which was a 10-fold increase in M2 cells (Fig. 3B). At the same time, addition of Box5 (a specific inhibitor of Wnt5a) completely reversed the pro-M2 function of Wnt5a (Fig. 3B).

A variety of cytokines are closely related to M2 macrophage polarization(13). Therefore, we screened several M2 polarization-associated cytokines As shown in Fig. 3C, RT-qPCR results showed that the expression of IL-10 was significantly increased after Wnt5a stimulation. Western blot and ELISA revealed that IL-10 protein expression and secretion level were both significantly elevated (Fig. 3D and E). Wnt5a-induced M2 polarization was almost completely suppressed by IL-10 antibody (Fig. 3F and G). Additionally, confocal immunofluorescence microscopy showed that Wnt5a, CD163 and IL-10 were frequently co-localized in CRC samples (Fig. 3H). Taken together, these results demonstrate that Wnt5a induces M2 macrophage polarization via IL-10.

\section{CaKMII-ERK1/2-STAT3 pathway is required for Wnt5a-induced IL-10 expression in macrophages}

To determine the molecular mechanism by which Wnt5a up-regulated IL-10, we looked at ERK1/2, STAT3 and NF-KB signaling pathways, of which all had been reported to be involved in the regulation of IL-10 expression and macrophage polarization(25, 29, 30). Western blot was carried out to estimate the phosphorylation status of ERK1/2, STAT3, and NF-KB in Wnt5a-treated macrophages. As shown in Fig. 4A, phosphorylation levels of ERK1/2 (p-ERK1/2) and STAT3 (p-STAT3) significantly increased after Wnt5a treatment, whereas there was no significant change in the phosphorylation level of p65 (an important component of NF-KB). Immunofluorescence analysis showed that both ERK1/2 and STAT3 translocated to the nucleus of Wnt5a-stimulated macrophages (Fig. 4B). To explore whether IL-10 were regulated by ERK1/2 and STAT3 pathway, U0126 (specific small-molecule inhibitor of p-ERK1/2) and S3I-201 (specific small-molecule inhibitor of p-STAT3) were used. Results suggested that blocking p-ERK1/2 with U0126 neutralized IL-10 induction by Wnt5a, as was the case with blocking p-STAT3 with S3I-201 (Fig. 4C). Moreover, U0126 not only suppressed p-ERK1/2 but also p-STAT3 expression, and S3I-201 hardly affected the expression of p-ERK1/2 (Fig. 4C). These results reveal that Wnt5a affects IL-10 expression through the ERK1/2 and STAT3 pathways, and STAT3 is phosphorylated by ERK1/2. In addition, U0126 or S3I-201 significantly reduced the ratio of the M2 cells in Wnt5a-treated macrophages (Fig. 4D). Consistently, the secretion level of IL-10 induced by Wnt5a is mostly abolished by U0126 or S3I-201(Fig. 4E).

$\mathrm{Ca}^{2+} / \mathrm{CaMKII}$ pathway is one of the important non-canonical pathways of Wnt5a signaling, which may further activate the downstream pathway of ERK1/2 $(17,31)$. Hence, we tested the expression level of $\mathrm{p}$-CaMKII in Wnt5a-stimulated macrophages. We found that Ca ${ }^{2+} / \mathrm{CaMKII} \mathrm{Pathway}$ was

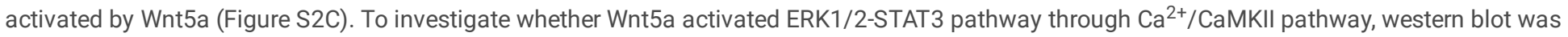
carried out to test p-ERK1/2 and p-STAT3 expression in Wnt5a-treated macrophages with or without CK59 (p-CaMKII inhibitor) or U0126 or S3I-201. Results showed that Wnt5a-induced up-regulation of p-ERK1/2 and p-STAT3 expression were entirely abolished by CK59, while p-CaMKII level was not affected by U0126 or S3I-201 (Fig. 4F). Meanwhile, CK59, U0126 and S3I-201 inhibited IL-10 protein expression (Fig. 4F). ELISA and flow cytometry analyses suggested that CK59 almost completely repressed IL-10 secretion level and the pro-M2 function of Wnt5a (Fig. $4 \mathrm{G}$ and $\mathrm{H}$ ). Collectively, the above results demonstrate that Wnt5a-CaMKII-ERK1/2-STAT3 pathway is crucial for IL-10-mediated M2 polarization. 
To evaluate the role of Wnt5a in CRC cells, sh-Wnt5a or sh-NC was transfected into SW480 cells and recombinant Wnt5a protein was given to treat HCT116 or DLD-1 cells directly. The transfection efficiency of sh-Wnt5a or sh-NC was measured by RT-qPCR (Figure S3A). The CCK-8 and colony formation assay analyses showed that tumor cell proliferation was not affected by knockdown of Wnt5a (Figure S3B and C), as well as tumor cell migration (Figure S3D). Similarly, Wnt5a hardly affected the proliferation and migration capability of HCT116 or DLD-1 cells (Figure S3E-J). These results indicate that Wnt5a does not directly influence the malignant biological behavior of CRC cells.

However, it is unclear whether Wnt5a affects the biological behavior of tumor cells through the crosstalk between TAMs and cancer cells. To clarify the functions of Wnt5a-induced macrophages in CRC in vitro, M0 macrophages were first treated with Wnt5a with or without IL-10 antibody or IgG for $72 \mathrm{~h}$, and then co-cultured with HCT116 or DLD-1 cells for another $36 \mathrm{~h}$, which were harvested for subsequent experiments (Fig. 5A). Clonogenic assay showed that M0 macrophages treated by Wnt5a obviously improved the clonogenic survival of HCT116 cells, as well as DLD-1 cells (Fig. 5B and C). After addition of IL-10 antibody antagonized the pro-M2 function of Wnt5a, this effect was reversed (Fig. 5B and C). The migration of cancer cells co-cultured with Wnt5a-induced M2 macrophages was significantly enhanced, which was reversed by IL-10 neutralizing antibody (Fig. $5 \mathrm{D}$ and $\mathrm{E})$. In the transwell invasion assay, the invasive ability of cancer cells co-cultured with Wnt5a-induced M2 macrophages was also enhanced, and this enhancement was suppressed by IL-10 neutralizing antibody (Fig. 5F and G). Taken together, these data reveal that M2 macrophages are critical for the cancer-promoting functions of Wnt5a.

\section{TAMs depend on Wnt5a for their cancer-promoting roles in vitro}

We further explored the role of Wnt5a in TAMs. After being treated with Box5 (Wnt5a inhibitor), the expression level of IL-10, p-CaMKII, p-ERK1/2 and p-STAT3 were all down-regulated in TAMs (Fig. 6A). Subsequently, we verified whether the CaKMII-ERK1/2-STAT3 regulatory pathway was also present in TAMs. As shown in Fig. 6B, U0126 suppressed the level of p-STAT3 and IL-10, whereas S3I-201 only reduced the expression of IL-10 without affecting p-ERK1/2 expression. IL-10, p-ERK1/2 and p-STAT3 expression were simultaneously repressed by CK59 (Fig. 6C), while the level of p-CaMKII was not affected by U0126 or S3I-201 (Fig. 6B). The secretion level of IL-10 in TAMs was also reduced under those inhibitors' treatment (Fig. 6D), as well as the proportion of M2 cells (Fig. 6E). These results indicate that Wnt5a is involved in the regulation of M2-like TAM polarization through CaKMII-ERK1/2-STAT3 pathway-mediated IL-10 secretion.

We then investigated the effect of Wnt5a+ TAMs on CRC cells. Lentiviral vectors expressing sh-Wnt5a or sh-NC were transfected into TAMs for further functional analysis in vitro. The transfection efficiency was measured by RT-qPCR and western blot (Figure S4 A and B). Clone formation assay showed that the proliferation capacity of CRC cells co-cultured with TAMs was significantly stronger than that co-cultured with sh-Wnt5a TAMs (Fig. 6F and G). Similarly, transwell assay suggested that the migration and invasion capacity of CRC cells were dramatically enhanced by TAMs, and this enhancement was obviously attenuated after knockdown of Wnt5a in TAMs (Fig. 6H-K). Together, our findings demonstrate that Wnt5a is crucial for TAM-mediated migration and invasion of cancer cells.

\section{Silencing Wnt5a inhibits the pro-tumor effect of TAMs in vivo}

To confirm the above in vitro results, an in vivo xenograft model was used. HCT116 cells alone, TAMs alone, HCT116 + sh-NC TAMs and HCT116 + sh-Wnt5a TAMs were injected subcutaneously into the right flank of female nude mice. After 45 days, tumors in the HCT116 + sh-NC TAMs group were significantly larger and heavier than those in the HCT116 + sh-Wnt5a TAMs and HCT116 group (Fig. 7A-C). No tumors were produced in the TAMs group (data not shown). Subsequently, RT-qPCR (Fig. 7D) and immunohistochemical staining (Fig. 7E) showed that in the group injected with HCT116 + sh-Wnt5a TAMs, Wnt5a, CD163 and IL-10 expression levels were significantly lower compared with the HCT116 + sh-NC TAMs group. The protein levels of Wnt5a, IL-10, p-CaMKII, p-ERK1/2, and p-STAT3 were significantly higher in the HCT116 + sh-NC TAMs group compared with the other two groups (Fig. 7F). Additionally, Ki67 staining, an indicator of tumor proliferation, also decreased in the HCT116 + sh-Wnt5a TAMs group compared with HCT116 + sh-NC TAMs group (Fig. 7G). These data indicate that sh-Wnt5a TAMs have a much lower tumor support capacity compared with sh-NC TAMs. It is essential for cancer cells to enter the blood vessel to become CTCs for distant metastasis. We then tested the presence of CTCs in peripheral blood of the mice. Representative CTC images were presented in Fig. 7H. Further counting analysis showed significant more CTCs were detected in the HCT116 + sh-NC TAM group compared with the other two groups (Fig. 7I). Liver/lung metastatic lesions were found in one out of six mice injected with HCT116 + sh-Wnt5a TAMs, none in mice injected with only HCT116 cells, while four in mice injected with HCT116 + sh-NC TAM (Fig. 7J and K). Collectively, these results demonstrate that TAMs enhance the growth and metastasis of CRC, whereas Wnt5a knockdown impairs the cancer-promoting functions of TAMs in vivo.

\section{Discussion}


In this study, we firstly found that high $\mathrm{Wnt} 5 \mathrm{a}^{+} \mathrm{CD} 68^{+} / \mathrm{CD} 68^{+} \mathrm{TAMs}$ ratio was significantly associated with clinicopathologic characteristics and poor prognosis in CRC patients. Further investigation confirmed that Wnt5a was involved in M2 polarization of TAMs by regulating CaMKII-ERK1/2STAT3 pathway-mediated IL-10 secretion, thereby promoting CRC progression.

Clinically, elevated Wnt5a expression in CRC was found to be mainly localized in TAMs. High Wnt5a ${ }^{+} \mathrm{TAMs}^{\mathrm{T}} \mathrm{TAMs}\left(\mathrm{Wnt5a}^{+} \mathrm{CD} 68^{+} / \mathrm{CD} 68^{+}\right)$ratio was significantly correlated with LVI, TI, LNM, TNM stage and poor prognosis in CRC patients. Previously, the association of TAMs with cancer prognosis had been extensively studied. Some studies showed that TAMs infiltration was a poor prognostic factor(32-34), while others reported that TAMs infiltration was associated with better prognosis(35-37). The discrepancy was especially pronounced in CRC(32, 35, 37). We noticed that CD68 was used in these studies to identify TAMs, which was generally considered to be a pan-macrophage marker, making this protein unspecific for distinguishing TAM subtypes and their different roles in CRC progression. On the other hand, TAM subtypes were found to be a better choice for predicting cancer prognosis. Numerous studies had shown that high $\mathrm{CD}_{163^{+}}$TAM subtype infiltration was significantly associated with poor clinical outcomes in numerous solid tumors $(8,38,39)$. Cortes et al. revealed that ZEB1 ${ }^{+}$TAM subtype was correlated with tumor growth, chemotherapy resistance and poor survival in ovarian carcinoma(40). In this work, we confirmed that Wnt5a ${ }^{+}$TAM subtype was a preferable indicator of CRC prognosis to TAM. Therefore, Wnt5a+ TAM subtype could serve as a poor prognostic maker for evaluating prognosis in CRC patents.

Functional polarization of TAMs to M1 or M2 represents a key mechanism that controls their functions, switching roles between tumor suppression and promotion $(10,41)$. Multiple studies tended to M2 TAMs, which were closely related to poor prognosis in $\mathrm{CRC}(8,42)$. We found that Wnt5a was co-localized with CD163 (M2 marker), rather than HLA-DR (M1 marker). We also found that Wnt5a was involved in inducing macrophage polarization to $\mathrm{M} 2$ phenotype. These results indicate that Wnt5a ${ }^{+}$TAM is an M2-like TAM subtype. Further in vitro and in vivo experiments confirmed that Wnt5a+ TAMs promoted CRC cells growth, migration, invasion and metastasis. Knocking down Wnt5a mostly impaired the protumor effect of TAMs, which revealed that TAMs depended on Wnt5a for their cancer-promoting roles. Intriguingly, knocking down Wnt5a did not alter the proliferation and migration capacity of SW480, and direct stimulation with recombinant Wnt5a protein also hardly affected the proliferation and migration ability of HCT116 or DLD-1 cells. These results reveal that Wnt5a does not directly influence CRC cells, but indirectly facilitates cancer progression by regulating TAM M2 polarization. In contrast, a few studies showed that Wnt5a had no or weak expression in colorectal cancer and mainly played a role in tumor suppression $(43,44)$. Their research focused on the direct effects of Wnt5a on cancer cells, while ours concentrated on the regulatory role of Wnt5a in TAMs. In fact, Smith and colleagues reported a long time ago that Wnt5a was mainly expressed in TAMs in CRC(24), which is consistent with our findings. In addition, Nitzki et al. also found that Wnt5a was mainly derived from the tumor stroma, especially from TAMs in basal cell carcinoma(23). Therefore, we inferred that Wnt5a was more likely to play a major role in altering the tumor microenvironment, regulating M2-like TAM polarization, inhibiting tumor immune response, and thus contributing to tumor invasion and metastasis. These data demonstrate that M2-like TAM is an important mediator of Wnt5a to promote CRC progression.

M2 polarization of macrophages is well known to be driven by multiple signaling pathways and cytokines including IL-4, IL-13 and IL-10(13, 45). In terms of cytokines, here we demonstrated that Wnt5a stimulated macrophages to produce IL-10, which in turn induced M2 polarization of macrophages, finally facilitating tumor growth, invasion and metastasis. This observation revealed that the protumorigenic effects of Wnt5a were mediated by IL-10-induced M2-like TAM polarization. Similarly, this way of regulating IL-10 expression was also observed in the study by Lee and colleagues. They stated that BMP-6 (bone morphogenetic proteins-6) stimulated TAMs to produce IL-10, which then induced M2 polarization of TAMs to mediate the pro-tumor effect of BMP-6(30). Mechanistically, we demonstrated that CaMKII-ERK1/2-STAT3 pathway was essential for IL-10 production induced by Wnt5a. It had been confirmed that STAT3 bound directly to the -588 to -800 bp region of IL-10 promoter to promote IL-10 transcription(30). Ma et al. found that dysregulation of CaMKII in intestinal epithelial cells regulated colitis-associated colorectal carcinogenesis by enhancing STAT3 activation(46). Unudurthi et al. showed that CaMKII was aberrantly activated in cardiac hypertrophy and heart failure, leading to degradation of $\beta I V$-spectrin, resulting in an increase of free STAT3 level(47). However, it was unclear in their work whether STAT3 was phosphorylated either by CaMKII or indirectly through another kinase. Zhang and colleagues reported that ERK1/2 activated the STAT3 pathway in the regulation of M2-like TAMs polarization(29). We also identified that CaMKII indirectly promoted STAT3 phosphorylation through the ERK1/2 pathway. On the whole, Wnt5a-CaMKII-ERK1/2-STAT3 pathway plays an important role in M2 polarization. Their specific regulatory mechanisms are still unclear, especially the regulation of STAT3 by ERK1/2. Further investigation is necessary to clarify the mechanism by which ERK1/2 regulates the STAT3 pathway.

In this work, we demonstrated that TAMs secreted both Wnt5a and IL-10, which formed a Wnt5a/IL-10 autocrine loop, resulting in M2 polarization of TAMs. Previously, multiple studies showed that Wnt5a was induced by inflammatory factors $(25,48)$. Inflammation microenvironment is a recognized hallmark of $\mathrm{CRC}(49)$, and CRC cells could also secrete a variety of inflammatory factors to promote tumor invasion and metastasis(50, 51). Therefore, the inflammatory factors in tumor microenvironment may play an important role in Wnt5a induction. Further exploration is required to elucidate the mechanism by which Wnt5a expression is up-regulated in TAMs.

M2 polarization of TAMs is a multi-factor, multi-step and complex pathological process in the tumor microenvironment(13). Recently, we found that IL-4 secreted by colorectal cancer cells affected M2 polarization of TAM(9). Moreover, factors such as GM-CSF(12) and exosomes(52) derived from cancer cells are involved in inducing macrophages M2 polarization. Therefore, many factors derived from cancer cells or tumor microenvironment 
could affect M2 polarization of macrophages during colorectal cancer progression. M2 macrophages are generally considered to inhibit inflammatory responses and promote tumor immune escape during tumor progression(6). In this study, we demonstrated that Wnt5a induced M2 macrophage polarization via IL-10. In contrast, Pereira et al. showed that Wnt5a was induced by LPS/IFN-Y and suppressed by IL-10 in macrophages(53). Their research was mainly based on changes in mRNA expression level of Wnt5a(53). However, Leandersson and colleagues showed that Wnt5a was regulated at the post-transcriptional level, and the mRNA expression level of Wnt5a is not closely related to its protein level, indicating that Wnt5a mRNA expression could not reflect its protein level(54). In addition, Pereira's study only emphasized the upregulation of Wnt5a expression in macrophages derived from sepsis patients, and did not further explore the effect of Wnt5a on macrophage differentiation(53). However, Bergenfelz et al. had confirmed that Wnt5a induced LPS/IFN-y-activated M1 macrophages to dedifferentiate into tolerogenic phenotype of macrophages via IL-10 in sepsis(25). Li and colleagues also found that Wnt5a inhibited inflammation-driven intervertebral disc degeneration through negative feedback regulation of NF-kB(55). Therefore, Wnt5a might be an important mediator to suppress inflammatory response of macrophages in a negative feedback manner.

\section{Conclusion}

In summary, our results revealed that, as an M2-like TAM subtype, high Wnt5a+ TAMs/CD68 ${ }^{+}$TAMs ratio was significantly associated with poor prognosis in CRC patients. Mechanistically, Wnt5a induced M2 polarization of TAMs by regulating CaMKII-ERK1/2-STAT3 pathway-mediated IL-10 secretion, thereby promoting tumor growth, invasion and metastasis of CRC. Our study highlighted that Wnt5a ${ }^{+}$TAM or Wnt5a/CaMKII/ERK/STAT3/IL-10 axis might be a novel potential immunotherapy target for combating CRC.

\section{Abbreviations}

TME: tumor microenvironment; TAMs: tumor-associated macrophages; EMT: Epithelial-mesenchymal transition; CRC: colorectal cancer; CTC: circulating tumor cell; Wnt5a: wingless-type MMTV integration site family, member 5a; IL-10: interleukin-10; RFS: recurrence-free survival; OS: overall survival; ERK: extracellular regulated protein kinases; STAT3: signal transducer and activator of transcription 3; CaMKII: calmodulindependent protein kinase II.

\section{Declarations}

\section{Ethics approval and consent to participate}

This study was endorsed by the ethics committee of Wuhan University and informed consents were obtained from all included patients. All animal experiments were performed strictly according to the guidelines of the Institutional Animal Care and Use Committee of the Zhongnan Hospital of Wuhan University.

\section{Consent for publication}

Not applicable

\section{Availability of data and materials}

The original data will be transferred and shared following the instruction by the Editorial Committee.

\section{Competing interests}

The authors declare that they have no competing interests.

\section{Funding}

This work was supported by grants from the National Natural Science Foundation of China (81572874; 81872376), National Natural Science Fund Youth Fund of China (81702411), and Health Commission of Hubei Province Scientific Research Project (WJ2019H012).

\section{Authors contributions}

QL designed the subject study and drafted the manuscript. CY, SW, DS, CW contributed to the study design and the manuscript. QL, CY, JS performed experiments. XL, RD collected clinical samples and clinicopathological parameters. QL, CY, SW, DS carried out the data analyses. All authors read and approved the final manuscript.

\section{Acknowledgments}

We are grateful to the excellent technical supports for CTCs isolation and identification provided by Song Han researcher from Wuhan YZY Medical Science and Technology Co., Ltd. (Wuhan, China). We are also particularly grateful to Jun Jiang for his contribution to the revision of the 
manuscript.

\section{References}

1. Wan L, Pantel K, Kang Y. Tumor metastasis: moving new biological insights into the clinic. Nat Med. 2013;19(11):1450-64.

2. Quail DF, Joyce JA. Microenvironmental regulation of tumor progression and metastasis. Nat Med. 2013;19(11):1423-37.

3. Lopez-Soto A, Gonzalez S, Smyth MJ, Galluzzi L. Control of Metastasis by NK Cells. Cancer Cell. 2017;32(2):135-54.

4. Maman S, Witz IP. A history of exploring cancer in context. Nat Rev Cancer. 2018;18(6):359-76.

5. Jiang Y, Li Y, Zhu B. T-cell exhaustion in the tumor microenvironment. Cell Death Dis. 2015;6:e1792.

6. Noy R, Pollard JW. Tumor-associated macrophages: from mechanisms to therapy. Immunity. 2014;41(1):49-61.

7. Condeelis J, Pollard JW. Macrophages: obligate partners for tumor cell migration, invasion, and metastasis. Cell. 2006;124(2):263-6.

8. Wei C, Yang C, Wang S, Shi D, Zhang C, Lin X, et al. Crosstalk between cancer cells and tumor associated macrophages is required for mesenchymal circulating tumor cell-mediated colorectal cancer metastasis. Mol Cancer. 2019;18(1):64.

9. Lin X, Wang S, Sun M, Zhang C, Wei C, Yang C, et al. miR-195-5p/NOTCH2-mediated EMT modulates IL-4 secretion in colorectal cancer to affect M2-like TAM polarization. J Hematol Oncol. 2019;12(1):20.

10. Ruffell B, Affara NI, Coussens LM. Differential macrophage programming in the tumor microenvironment. Trends Immunol. 2012;33(3):119-26.

11. Chen Y, Zhang S, Wang Q, Zhang X. Tumor-recruited M2 macrophages promote gastric and breast cancer metastasis via M2 macrophagesecreted CHI3L1 protein. J Hematol Oncol. 2017;10(1):36.

12. Su S, Liu Q, Chen J, Chen J, Chen F, He C, et al. A positive feedback loop between mesenchymal-like cancer cells and macrophages is essential to breast cancer metastasis. Cancer Cell. 2014;25(5):605-20.

13. Mantovani A, Sozzani S, Locati M, Allavena P, Sica A. Macrophage polarization: tumor-associated macrophages as a paradigm for polarized M2 mononuclear phagocytes. Trends Immunol. 2002;23(11):549-55.

14. Ye YC, Zhao JL, Lu YT, Gao CC, Yang Y, Liang SQ, et al. NOTCH Signaling via WNT Regulates the Proliferation of Alternative, CCR2-Independent Tumor-Associated Macrophages in Hepatocellular Carcinoma. Cancer Res. 2019;79(16):4160-72.

15. Yang Y, Ye YC, Chen Y, Zhao JL, Gao CC, Han H, et al. Crosstalk between hepatic tumor cells and macrophages via Wnt/beta-catenin signaling promotes M2-like macrophage polarization and reinforces tumor malignant behaviors. Cell Death Dis. 2018;9(8):793.

16. Chae WJ, Bothwell ALM. Canonical and Non-Canonical Wnt Signaling in Immune Cells. Trends Immunol. 2018;39(10):830-47.

17. Asem MS, Buechler S, Wates RB, Miller DL, Stack MS. Wnt5a Signaling in Cancer. Cancers (Basel). 2016;8(9).

18. Chirumbolo S, Bjorklund G. Can Wnt5a and Wnt non-canonical pathways really mediate adipocyte de-differentiation in a tumour microenvironment? Eur J Cancer. 2016;64:96-100.

19. Valencia J, Hernandez-Lopez C, Martinez VG, Hidalgo L, Zapata AG, Vicente A, et al. Wnt5a skews dendritic cell differentiation to an unconventional phenotype with tolerogenic features. J Immunol. 2011;187(8):4129-39.

20. Feng Y, Liang Y, Zhu X, Wang M, Gui Y, Lu Q, et al. The signaling protein Wnt5a promotes TGFbeta1-mediated macrophage polarization and kidney fibrosis by inducing the transcriptional regulators Yap/Taz. J Biol Chem. 2018;293(50):19290-302.

21. Ziegler S, Gartner K, Scheuermann U, Zoeller T, Hantzschmann J, Over B, et al. Ca(2+) -related signaling events influence TLR9-induced IL-10 secretion in human B cells. Eur J Immunol. 2014;44(5):1285-98.

22. Pukrop T, Klemm F, Hagemann T, Gradl D, Schulz M, Siemes S, et al. Wnt 5a signaling is critical for macrophage-induced invasion of breast cancer cell lines. Proc Natl Acad Sci U S A. 2006;103(14):5454-9.

23. Nitzki F, Zibat A, Konig S, Wijgerde M, Rosenberger A, Brembeck FH, et al. Tumor stroma-derived Wnt5a induces differentiation of basal cell carcinoma of Ptch-mutant mice via CaMKII. Cancer Res. 2010;70(7):2739-48.

24. Smith K, Bui TD, Poulsom R, Kaklamanis L, Williams G, Harris AL. Up-regulation of macrophage wnt gene expression in adenoma-carcinoma progression of human colorectal cancer. Br J Cancer. 1999;81(3):496-502.

25. Bergenfelz C, Medrek C, Ekstrom E, Jirstrom K, Janols H, Wullt M, et al. Wnt5a induces a tolerogenic phenotype of macrophages in sepsis and breast cancer patients. J Immunol. 2012;188(11):5448-58.

26. Zhang B, Ye H, Ren X, Zheng S, Zhou Q, Chen C, et al. Macrophage-expressed CD51 promotes cancer stem cell properties via the TGFbeta1/smad2/3 axis in pancreatic cancer. Cancer Lett. 2019;459:204-15.

27. Chen FF, Wang SY, Fang Y, Zheng L, Zhi X, Cheng BR, et al. Feasibility of a novel one-stop ISET device to capture CTCs and its clinical application. Oncotarget. 2017;8(2):3029-41.

28. Huang YJ, Yang CK, Wei PL, Huynh TT, Whang-Peng J, Meng TC, et al. Ovatodiolide suppresses colon tumorigenesis and prevents polarization of M2 tumor-associated macrophages through YAP oncogenic pathways. J Hematol Oncol. 2017;10(1):60. 
29. Zhang G, Guo L, Yang C, Liu Y, He Y, Du Y, et al. A novel role of breast cancer-derived hyaluronan on inducement of M2-like tumor-associated macrophages formation. Oncoimmunology. 2016;5(6):e1172154.

30. Lee JH, Lee GT, Woo SH, Ha YS, Kwon SJ, Kim WJ, et al. BMP-6 in renal cell carcinoma promotes tumor proliferation through IL-10-dependent M2 polarization of tumor-associated macrophages. Cancer Res. 2013;73(12):3604-14.

31. Zhong W, Chebolu S, Darmani NA. Thapsigargin-induced activation of $\mathrm{Ca}(2+)$-CaMKII-ERK in brainstem contributes to substance P release and induction of emesis in the least shrew. Neuropharmacology. 2016;103:195-210.

32. Hamm A, Prenen H, Van Delm W, Di Matteo M, Wenes M, Delamarre E, et al. Tumour-educated circulating monocytes are powerful candidate biomarkers for diagnosis and disease follow-up of colorectal cancer. Gut. 2016;65(6):990-1000.

33. Yuan ZY, Luo RZ, Peng RJ, Wang SS, Xue C. High infiltration of tumor-associated macrophages in triple-negative breast cancer is associated with a higher risk of distant metastasis. Onco Targets Ther. 2014;7:1475-80.

34. Ryder M, Ghossein RA, Ricarte-Filho JC, Knauf JA, Fagin JA. Increased density of tumor-associated macrophages is associated with decreased survival in advanced thyroid cancer. Endocr Relat Cancer. 2008;15(4):1069-74.

35. Funada Y, Noguchi T, Kikuchi R, Takeno S, Uchida Y, Gabbert HE. Prognostic significance of CD8+ T cell and macrophage peritumoral infiltration in colorectal cancer. Oncol Rep. 2003;10(2):309-13.

36. Forssell J, Oberg A, Henriksson ML, Stenling R, Jung A, Palmqvist R. High macrophage infiltration along the tumor front correlates with improved survival in colon cancer. Clin Cancer Res. 2007;13(5):1472-9.

37. Braster R, Bogels M, Beelen RH, van Egmond M. The delicate balance of macrophages in colorectal cancer; their role in tumour development and therapeutic potential. Immunobiology. 2017;222(1):21-30.

38. He KF, Zhang L, Huang CF, Ma SR, Wang YF, Wang WM, et al. CD163+ tumor-associated macrophages correlated with poor prognosis and cancer stem cells in oral squamous cell carcinoma. Biomed Res Int. 2014;2014:838632.

39. Park JY, Sung JY, Lee J, Park YK, Kim YW, Kim GY, et al. Polarized CD163+ tumor-associated macrophages are associated with increased angiogenesis and CXCL12 expression in gastric cancer. Clin Res Hepatol Gastroenterol. 2016;40(3):357-65.

40. Cortes M, Sanchez-Moral L, de Barrios O, Fernandez-Acenero MJ, Martinez-Campanario MC, Esteve-Codina A, et al. Tumor-associated macrophages (TAMs) depend on ZEB1 for their cancer-promoting roles. EMBO J. 2017;36(22):3336-55.

41. Schmieder A, Michel J, Schonhaar K, Goerdt S, Schledzewski K. Differentiation and gene expression profile of tumor-associated macrophages. Semin Cancer Biol. 2012;22(4):289-97.

42. Vinnakota K, Zhang Y, Selvanesan BC, Topi G, Salim T, Sand-Dejmek J, et al. M2-like macrophages induce colon cancer cell invasion via matrix metalloproteinases. J Cell Physiol. 2017;232(12):3468-80.

43. Cheng R, Sun B, Liu Z, Zhao X, Qi L, Li Y, et al. Wnt5a suppresses colon cancer by inhibiting cell proliferation and epithelial-mesenchymal transition. J Cell Physiol. 2014;229(12):1908-17.

44. Dejmek J, Dejmek A, Safholm A, Sjolander A, Andersson T. Wnt-5a protein expression in primary dukes B colon cancers identifies a subgroup of patients with good prognosis. Cancer Res. 2005;65(20):9142-6.

45. Sica A, Mantovani A. Macrophage plasticity and polarization: in vivo veritas. J Clin Invest. 2012;122(3):787-95.

46. Ma X, Meng Z, Jin L, Xiao Z, Wang X, Tsark WM, et al. CAMK2gamma in intestinal epithelial cells modulates colitis-associated colorectal carcinogenesis via enhancing STAT3 activation. Oncogene. 2017;36(28):4060-71.

47. Unudurthi SD, Nassal D, Greer-Short A, Patel N, Howard T, Xu X, et al. betalV-Spectrin regulates STAT3 targeting to tune cardiac response to pressure overload. J Clin Invest. 2018;128(12):5561-72.

48. Rauner M, Stein N, Winzer M, Goettsch C, Zwerina J, Schett G, et al. WNT5A is induced by inflammatory mediators in bone marrow stromal cells and regulates cytokine and chemokine production. J Bone Miner Res. 2012;27(3):575-85.

49. Lasry A, Zinger A, Ben-Neriah Y. Inflammatory networks underlying colorectal cancer. Nat Immunol. 2016;17(3):230-40.

50. Hai Ping P, Feng Bo T, Li L, Nan Hui Y, Hong Z. IL-1beta/NF-kb signaling promotes colorectal cancer cell growth through miR-181a/PTEN axis. Arch Biochem Biophys. 2016;604:20-6.

51. Ye K, Chen QW, Sun YF, Lin JA, Xu JH. Loss of BMI-1 dampens migration and EMT of colorectal cancer in inflammatory microenvironment through TLR4/MD-2/MyD88-mediated NF-kappaB signaling. J Cell Biochem. 2018;119(2):1922-30.

52. Wang X, Luo G, Zhang K, Cao J, Huang C, Jiang T, et al. Hypoxic Tumor-Derived Exosomal miR-301a Mediates M2 Macrophage Polarization via PTEN/PI3Kgamma to Promote Pancreatic Cancer Metastasis. Cancer Res. 2018;78(16):4586-98.

53. Pereira C, Schaer DJ, Bachli EB, Kurrer MO, Schoedon G. Wnt5A/CaMKII signaling contributes to the inflammatory response of macrophages and is a target for the antiinflammatory action of activated protein C and interleukin-10. Arterioscler Thromb Vasc Biol. 2008;28(3):504-10.

54. Leandersson K, Riesbeck K, Andersson T. Wnt-5a mRNA translation is suppressed by the Elav-like protein HuR in human breast epithelial cells. Nucleic Acids Res. 2006;34(14):3988-99.

55. Li Z, Zhang K, Li X, Pan H, Li S, Chen F, et al. Wnt5a suppresses inflammation-driven intervertebral disc degeneration via a TNF-alpha/NFkappaB-Wnt5a negative-feedback loop. Osteoarthritis Cartilage. 2018;26(7):966-77.

Page $11 / 20$ 


\section{Tables}

Table 1 Correlation between the ratio of $\mathrm{Wnt5} \mathrm{a}^{+} \mathrm{CD} 68^{+} / \mathrm{CD} 68^{+}$macrophages and clinicopathologic parameters $(\mathrm{n}=63)$.

\begin{tabular}{|c|c|c|c|c|c|}
\hline \multirow[t]{2}{*}{ Parameters } & \multirow[t]{2}{*}{ n (\%) } & \multicolumn{2}{|c|}{$\mathrm{Wnt5a}^{+} \mathrm{CD} 8^{+} / \mathrm{CD} 68^{+}$ratio $(\mathrm{n})$} & \multirow[t]{2}{*}{$c^{2}$ value } & \multirow[t]{2}{*}{$P$ value } \\
\hline & & Low $(n=32)$ & High $(n=31)$ & & \\
\hline Gender & & & & 0.024 & 0.877 \\
\hline Male & $38(60.32)$ & 19 & 19 & & \\
\hline Female & $25(39.68)$ & 13 & 12 & & \\
\hline Age (years) & & & & 1.905 & 0.167 \\
\hline$\square 60$ & $29(46.03)$ & 12 & 17 & & \\
\hline$\geq 60$ & $34(53.97)$ & 20 & 14 & & \\
\hline Tumor site & & & & 0.013 & 0.910 \\
\hline Colon & $35(55.56)$ & 18 & 17 & & \\
\hline Rectum & $28(44.44)$ & 14 & 14 & & \\
\hline Tumor size $(\mathrm{cm})$ & & & & 0.386 & 0.535 \\
\hline$\square 5$ & $41(65.08)$ & 22 & 19 & & \\
\hline$\geq 5$ & $22(34.92)$ & 10 & 12 & & \\
\hline Tumor grade & & & & 1.932 & 0.165 \\
\hline Poor & $38(60.32)$ & 22 & 16 & & \\
\hline Moderate/Well & $25(39.68)$ & 10 & 15 & & \\
\hline LVI & & & & 5.773 & 0.016 \\
\hline Absence & $30(47.62)$ & 20 & 10 & & \\
\hline Presence & $33(52.38)$ & 12 & 21 & & \\
\hline PNI & & & & 1.905 & 0.167 \\
\hline Absence & 34 (53.97) & 20 & 14 & & \\
\hline Presence & $29(46.03)$ & 12 & 17 & & \\
\hline $\mathrm{TI}$ & & & & 6.719 & 0.010 \\
\hline T1-2 & $15(23.81)$ & 12 & 3 & & \\
\hline T3-4 & $48(76.19)$ & 20 & 28 & & \\
\hline LNM & & & & 7.014 & 0.008 \\
\hline N0-1 & $31(49.21)$ & 21 & 10 & & \\
\hline N2-3 & $32(50.79)$ & 11 & 21 & & \\
\hline TNM stage $\#$ & & & & 9.908 & 0.002 \\
\hline $\mathrm{I} / \mathrm{II}$ & $33(52.38)$ & 23 & 10 & & \\
\hline III & $30(47.62)$ & 9 & 21 & & \\
\hline CEA (ng/ml) & & & & 0.128 & 0.721 \\
\hline$\square 5$ & $44(69.84)$ & 23 & 21 & & \\
\hline$\geq 5$ & $19(30.16)$ & 9 & 10 & & \\
\hline
\end{tabular}

Notes: Bold indicates $P<0.05$; \#The 7th edition of the AJCC Cancer Staging Manual. Abbreviations: LVI, lymphovascular invasion; PNI, perineural invasion; TI, tumor invasion; LNM, lymph node metastasis; TNM, tumor-node-metastasis; CEA, carcinoembryonic antigen; CD68, cluster of differentiation 68@Wnt5a, wingless-type MMTV integration site family, member 5a. 
Table 2 Univariate and multivariate analyses of clinicopathologic parameters associated with recurrence-free survival and overall survival.

\begin{tabular}{|c|c|c|c|c|c|c|c|c|c|c|c|c|}
\hline \multirow[t]{3}{*}{ Parameters } & \multicolumn{6}{|c|}{ Recurrence-free survival } & \multicolumn{6}{|c|}{ Overall survival } \\
\hline & \multicolumn{3}{|c|}{ Univariate analysis } & \multicolumn{3}{|c|}{ Multivariate analysis } & \multicolumn{3}{|c|}{ Univariate analysis } & \multicolumn{3}{|c|}{ Multivariate analysis } \\
\hline & HR & $\begin{array}{c}95 \% \\
\text { CI }\end{array}$ & $P$ & HR & $95 \% \mathrm{CI}$ & $P$ & HR & $\begin{array}{c}95 \% \\
\text { CI }\end{array}$ & $P$ & $\mathrm{HR}$ & $\begin{array}{c}95 \% \\
\text { CI }\end{array}$ & $P$ \\
\hline Gender (Male vs. & & $0.391-$ & & & & & & $0.321-$ & & & & \\
\hline Female) & 0.782 & 1.564 & 0.488 & & & & 0.67 & 1.400 & 0.287 & & & \\
\hline $\begin{array}{l}\text { Age ( } \geq 60 \text { years vs. } \\
60 \text { years })\end{array}$ & 0.639 & $\begin{array}{l}0.325- \\
1.255\end{array}$ & 0.194 & & & & 0.641 & $\begin{array}{l}0.316- \\
1.303\end{array}$ & 0.219 & & & \\
\hline $\begin{array}{l}\text { Tumor site (Colon vs. } \\
\text { Rectum) }\end{array}$ & 1.015 & $\begin{array}{l}0.515- \\
2.001\end{array}$ & 0.965 & & & & 1.418 & $\begin{array}{l}0.700- \\
2.876\end{array}$ & 0.332 & & & \\
\hline $\begin{array}{l}\text { Tumor size }(\square 5 \mathrm{~cm} \\
\text { vs. } \geq 5 \mathrm{~cm})\end{array}$ & 1.255 & $\begin{array}{l}0.627- \\
2.512\end{array}$ & 0.521 & & & & 1.474 & $\begin{array}{l}0.721- \\
3.016\end{array}$ & 0.288 & & & \\
\hline $\begin{array}{l}\text { Tumor grade (Well vs } \\
\text { Moderate vs Poor) }\end{array}$ & 1.755 & $\begin{array}{l}0.894- \\
3.446\end{array}$ & 0.102 & & & & 1.263 & $\begin{array}{l}0.623- \\
2.564\end{array}$ & 0.517 & & & \\
\hline $\begin{array}{l}\text { LVI (Absence vs. } \\
\text { Presence) }\end{array}$ & 2.134 & $\begin{array}{l}1.051- \\
4.333\end{array}$ & 0.036 & 1.998 & $\begin{array}{l}0.937- \\
4.257\end{array}$ & 0.073 & 2.51 & $\begin{array}{l}1.178- \\
5.350\end{array}$ & 0.017 & 2.336 & $\begin{array}{l}1.040- \\
5.251\end{array}$ & 0.040 \\
\hline $\begin{array}{l}\text { PI (Absence vs. } \\
\text { Presence) }\end{array}$ & 1.557 & $\begin{array}{l}0.793- \\
3.059\end{array}$ & 0.198 & & & & 1.955 & $\begin{array}{l}0.957- \\
3.997\end{array}$ & 0.066 & & & \\
\hline TI (T1-2 vs. T3-4) & 2.288 & $\begin{array}{l}1.299- \\
4.029\end{array}$ & 0.004 & 1.335 & $\begin{array}{l}0.686- \\
2.597\end{array}$ & 0.394 & 1.926 & $\begin{array}{l}1.094- \\
3.390\end{array}$ & 0.023 & 1.104 & $\begin{array}{l}0.562- \\
2.168\end{array}$ & 0.774 \\
\hline LNM (N0-1 vs. N2-3) & 1.464 & $\begin{array}{l}1.070- \\
2.002\end{array}$ & 0.017 & 0.648 & $\begin{array}{l}0.366- \\
1.147\end{array}$ & 0.136 & 1.387 & $\begin{array}{l}1.001- \\
1.921\end{array}$ & 0.049 & 0.630 & $\begin{array}{l}0.348- \\
1.139\end{array}$ & 0.126 \\
\hline $\begin{array}{l}\text { TNM stage }{ }^{\#} \text { (I vs. II } \\
\text { vs. III) }\end{array}$ & 3.423 & $\begin{array}{l}1.702- \\
6.887\end{array}$ & 0.001 & 5.133 & $\begin{array}{l}1.246- \\
21.156\end{array}$ & 0.024 & 2.884 & $\begin{array}{l}1.428- \\
5.823\end{array}$ & 0.003 & 4.693 & $\begin{array}{l}1.101- \\
20.011\end{array}$ & 0.037 \\
\hline $\begin{array}{l}\text { CEA }(\geq 5 \mathrm{ng} / \mathrm{ml} \text { vs. } \\
5 \mathrm{ng} / \mathrm{ml})\end{array}$ & 0.791 & $\begin{array}{l}0.377- \\
1.657\end{array}$ & 0.533 & & & & 0.529 & $\begin{array}{l}0.227- \\
1.231\end{array}$ & 0.14 & & & \\
\hline $\begin{array}{l}\text { CD68 }{ }^{+} \text {macrophage } \\
\text { (Low vs. High) }\end{array}$ & 1.785 & $\begin{array}{l}0.901- \\
3.537\end{array}$ & 0.097 & & & & 1.465 & $\begin{array}{l}0.722- \\
2.975\end{array}$ & 0.29 & & & \\
\hline $\begin{array}{l}\text { Wnt5a }{ }^{+} \text {CD68 } 8^{+} \\
\text {macrophage (Low vs. } \\
\text { High) }\end{array}$ & 2.204 & $\begin{array}{l}1.088- \\
4.465\end{array}$ & 0.028 & 0.981 & $0.441-2.182$ & 0.962 & 2.184 & $\begin{array}{l}1.045- \\
4.563\end{array}$ & 0.038 & 0.925 & $\begin{array}{l}0.404- \\
2.115\end{array}$ & 0.853 \\
\hline $\begin{array}{l}\text { Wnt5a }{ }^{+} \mathrm{CD} 68^{+} / \mathrm{CD} 68^{+} \\
\text {macrophage (Low vs. } \\
\text { High) }\end{array}$ & 4.596 & $\begin{array}{l}2.126- \\
9.935\end{array}$ & $<0.001$ & 2.698 & $\begin{array}{l}1.110- \\
6.559\end{array}$ & 0.029 & 4.347 & $\begin{array}{l}1.934- \\
9.772\end{array}$ & $<0.001$ & 2.809 & $\begin{array}{l}1.107- \\
7.128 \\
\end{array}$ & 0.030 \\
\hline
\end{tabular}

Notes: Bold indicates $P<0.05$; \#The 7th edition of the AJCC Cancer Staging Manual. Abbreviations: LVI, lymphovascular invasion; PNI, perineural invasion; TI, tumor invasion; LNM, lymph node metastasis; TNM, tumor-node-metastasis; CEA, carcinoembryonic antigen; CD68, cluster of differentiation 68; Wnt5a, wingless-type MMTV integration site family, member 5a.

\section{Figures}



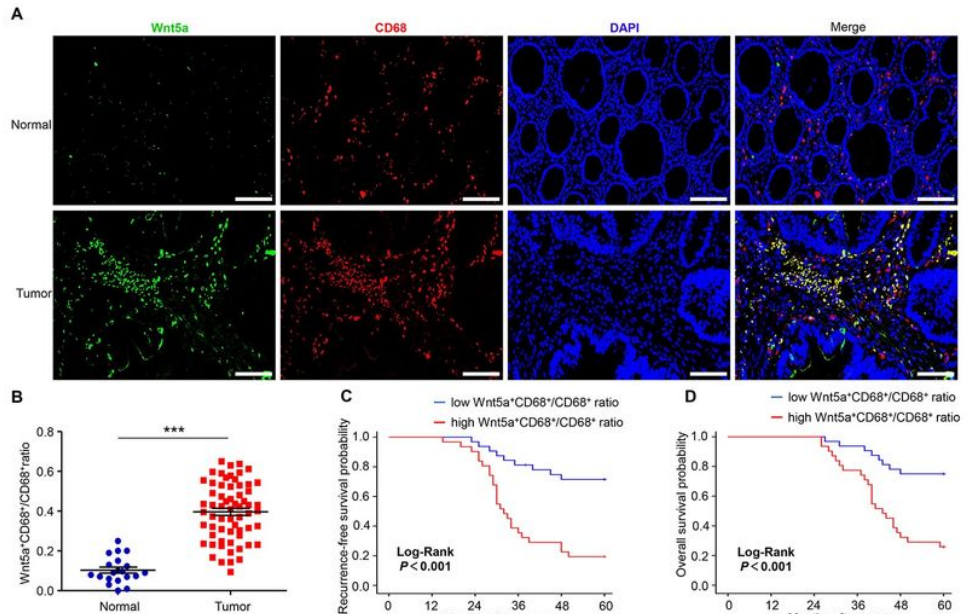

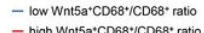
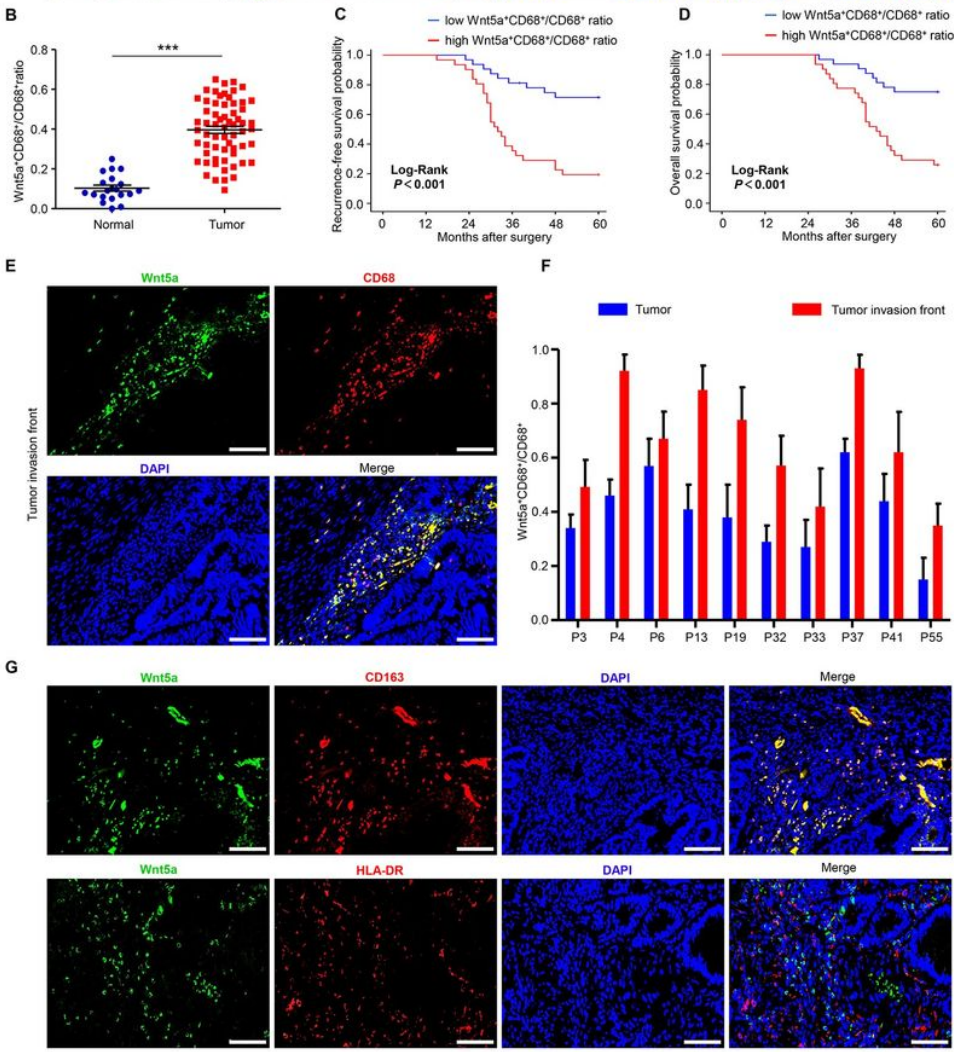

\section{Figure 1}

The ratio of Wnt5a+CD68+/CD68+ TAMs is correlated with poor prognosis in CRC patients. a Representative immunofluorescence staining images for Wnt5a (green), CD68 (red), DAPI (blue) in CRC samples. Bar $=100 \mu \mathrm{m}$. b Wnt5a+CD68+/CD68+ TAMs ratio was significantly elevated in primary human CRC tissues compared with normal colorectal tissues. Statistical analysis was conducted using one-way ANOVA. c, d Association of Wnt5a+CD68+/CD68+ TAMs ratio with recurrence-free survival and overall survival of CRC patients. e Representative immunofluorescence staining images for Wnt5a (green), CD68 (red), DAPI (blue) at tumor invasive front. Bar $=100 \mu \mathrm{m}$. f Wnt5a+CD68+/CD68+ TAMs ratio at tumor invasive front and tumor nest in $10 \mathrm{CRC}$ samples. g Representative immunofluorescence photographs for co-localization staining of Wnt5a, M2 maker (CD163) and M1 maker (HLA-DR). Bar $=100 \mu \mathrm{m}$. Error bars, SEM. ${ }^{* *} \mathrm{P}<0.001$ 

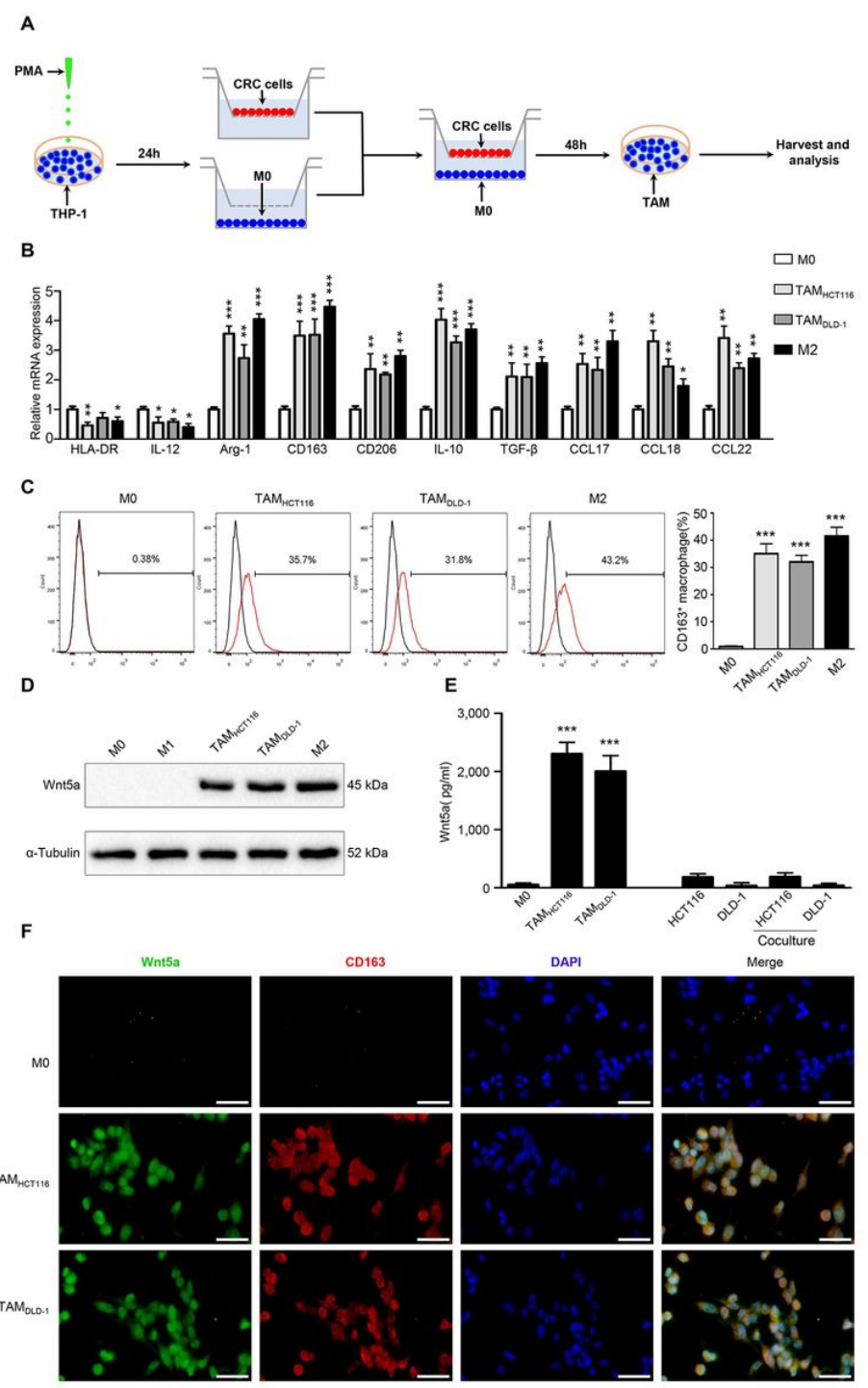

Figure 2

Wnt5a is mainly expressed in M2-like TAMs. a Flow chart of generating TAMs. b Relative expression of M1 markers (HLA-DR, IL-12), M2 markers (Arg-1, CD163, CD206, IL-10, TGFß, CCL17, CCL18, CCL22) in M0 macrophages, M2 macrophages and TAMs co-Cultured with HCT116 or DLD-1 for 48 hours. Error bars, SEM. c Flow cytometry analysis of the proportion of M2 cells in different groups of macrophages. Error bars, SEM. $d$ The expression level of Wnt5a in M0, M1, M2 macrophages and TAMs co-cultured with HCT116 or DLD-1 for 48 hours. e ELISA analysis of Wnt5a secretion level in macrophages, CRC cell lines and CRC cell lines co-cultured with macrophages. Error bars, SEM. $f$ Representative immunofluorescence photographs for Wnt5a, CD163 and DAPI in different groups of macrophages. Bar $=50 \mu \mathrm{m}$. All experiments were performed independently at least three times. Statistical analysis was conducted using one-way ANOVA. ${ }^{*} P<0.05$. ${ }^{* \star P}<<0.01$. ${ }^{* \star *} P<0.001$ 
A

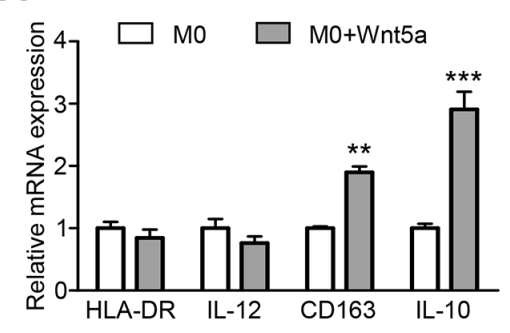

C

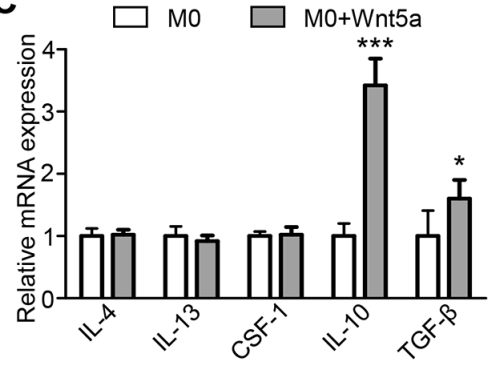

B

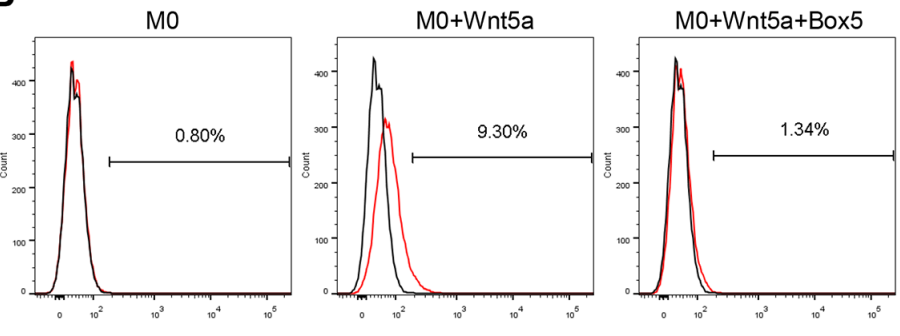

D

E

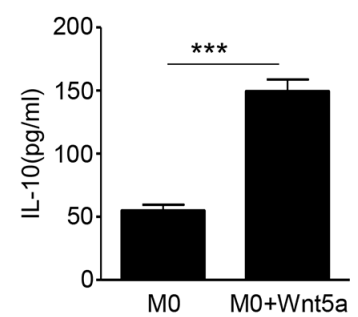

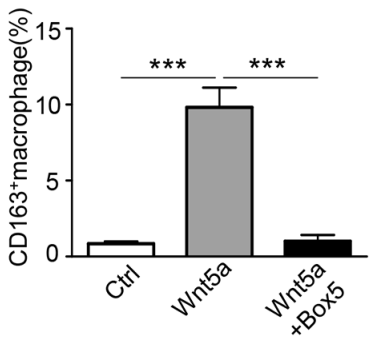

G

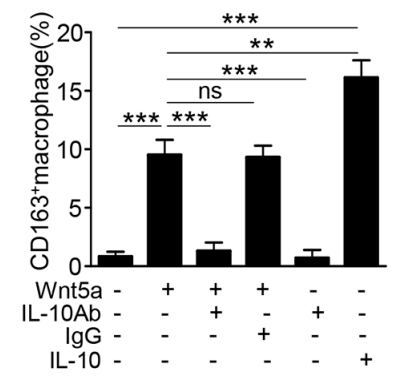

$\mathrm{MO+IL-10}$
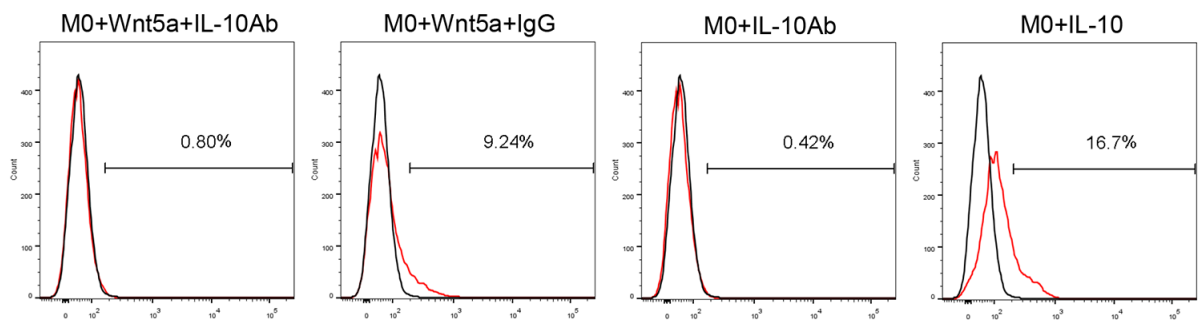

H
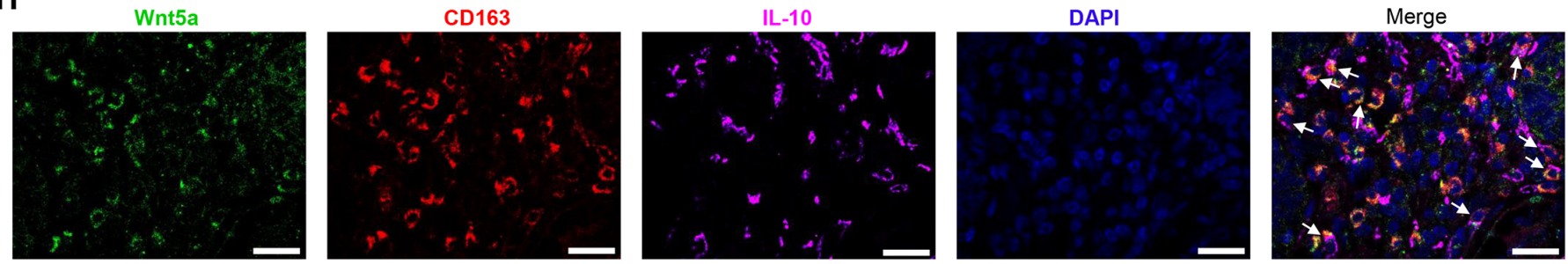

Figure 3

Wnt5a induces M2 macrophages polarization via IL-10. a RT-qPCR analysis of the relative expression levels of M1 markers (HLA-DR, IL-12) and M2 markers (CD163, IL-10) in M0 macrophages treated with Wnt5a. b Flow cytometry analysis showed M2 polarization (CD163+) in M0 macrophages treated with Wnt5a. This M2 polarization was reversed by Box5 (Wnt5a inhibitor). c RT-qPCR analysis of M2 polarization-associated cytokines expression in M0 macrophages with Wnt5a treatment. d Western blot analyzed the protein expression of IL-10 in Wnt5a-treated M0 macrophages. e ELISA analysis for IL10 secretion level in M0 macrophages treated with Wnt5a. f, g Flow cytometry analysis of M2 cells ratio in Wnt5a-stimulated M0 macrophages with or without IL-10 antibody or IgG or recombinant IL10 protein. h Confocal immunofluorescence analysis showed the colocalization expression of Wnt5a, CD163 and IL-10 in CRC sample. Bar $=25 \mu \mathrm{m}$. All experiments were performed independently at least three times. Statistical analysis was conducted using one-way ANOVA. Error bars, SEM. ns, not significant. ${ }^{*} P<0.05$. ${ }^{* * P<0.01}$. ${ }^{* \star * P} P<0.001$ 


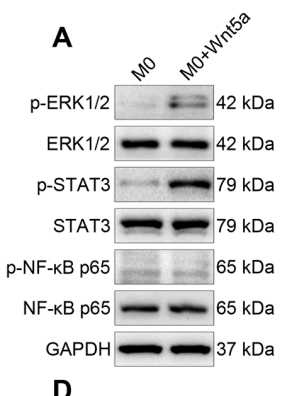

B

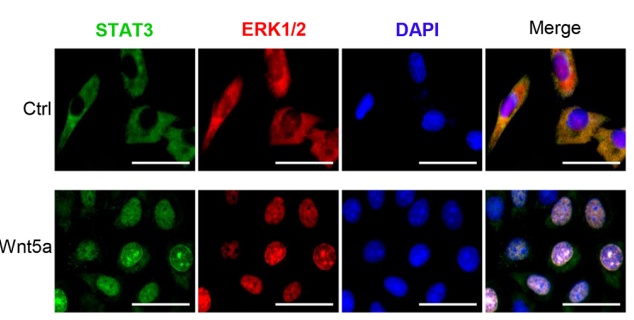

D
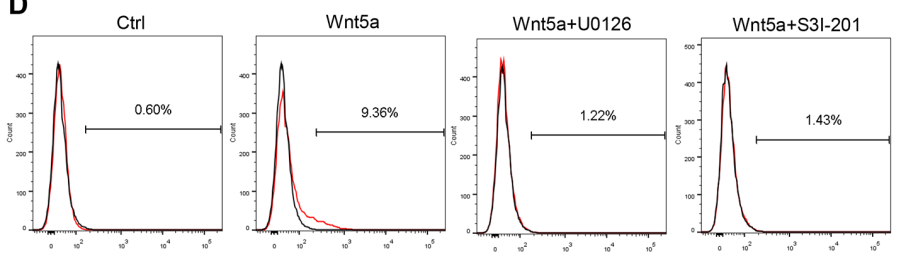

E

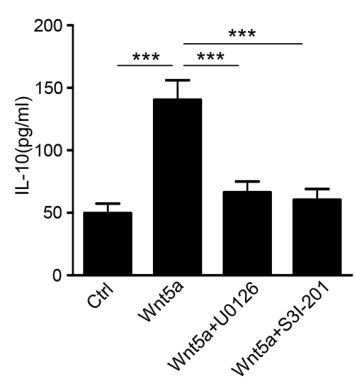

H

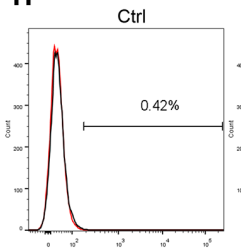

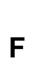

F

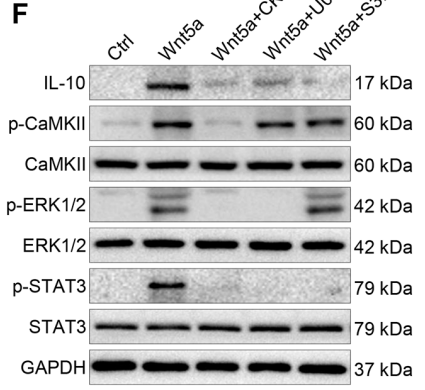

Wnt5a

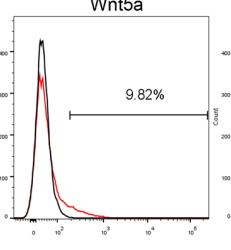

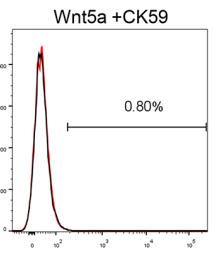

C
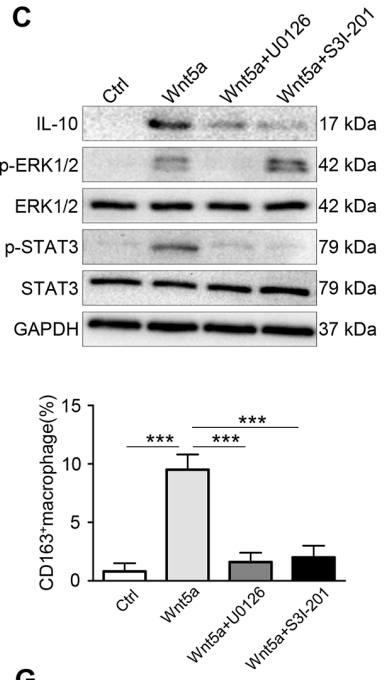

G

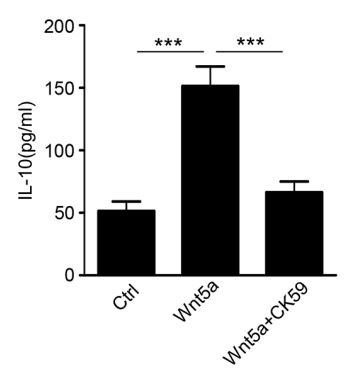

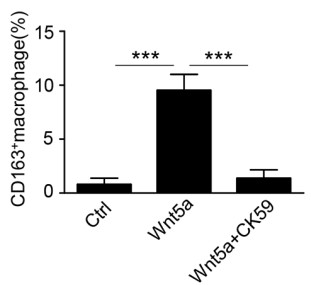

Figure 4

Wnt5a up-regulates IL-10 expression by activating CaKMII-ERK1/2-STAT3 pathway. a Western blot analysis of p-ERK1/2, p-STAT3 and p-NF-KB p65 expression in M0 macrophages with or without Wnt5a. b Immunofluorescence staining for ERK1/2 and STAT3 in M0 macrophages with or without Wnt5a. Bar $=5025 \mu \mathrm{m}$. c Western blot analysis of IL-10, p-ERK1/2 and p-STAT3 expression in Wnt5a-treated M0 macrophages with or without U0126 or S3I-201. d Flow cytometry analysis of Wnt5a-treated M0 macrophages with or without U0126 or S3I-201. Error bars, SEM. e ELISA analysis of IL-10 secretion level in Wnt5a-treated M0 macrophages with or without U0126 or S3I-201. Error bars, SEM. $f$ Western blot analysis of Wnt5a-treated M0 macrophages with or without CK59 or U0126 or S3I-201. g ELISA analysis of IL-10 secretion level in Wnt5a-treated M0 macrophages with or without CK59. Error bars, SEM. h Flow cytometry analysis of Wnt5a-treated M0 macrophages with or without CK59. Error bars, SEM. All experiments were performed independently at least three times. Statistical analysis was conducted using one-way ANOVA. *** $P<0.001$ 
A

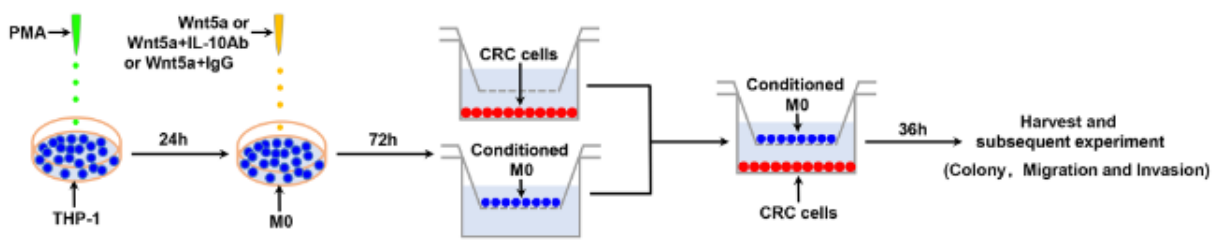

B

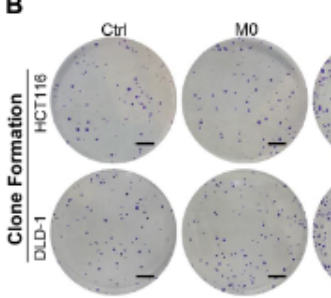

D

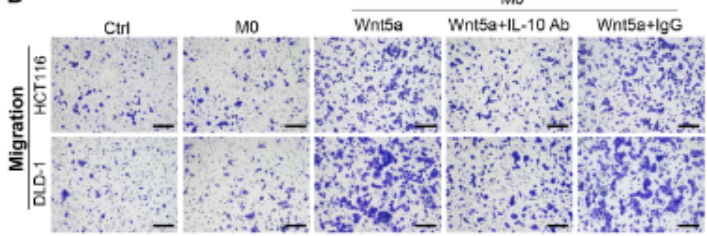

$\mathbf{F}$

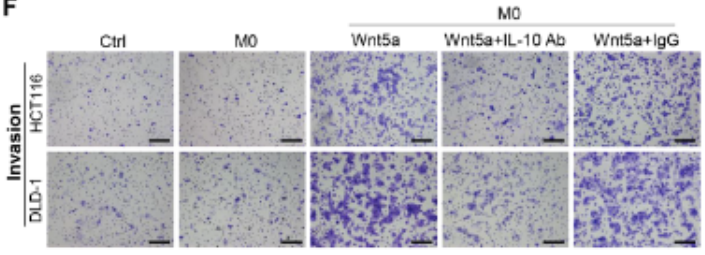

C

마rl ㅁ MO $\square$ Wnt5a-treated MO - Wnt5a+1L-10 Ab-treated M0 口Wnt5a+lgG-treated M0

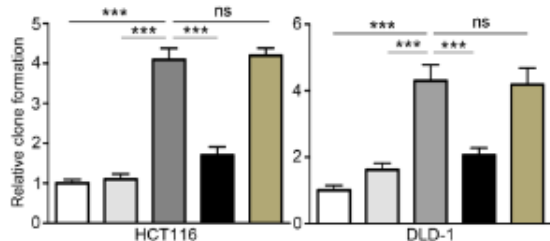

E $\square$ Ctrl $\square$ MO $\square$ Wnt5a-treated MO - Wnt5a+LL-10 Ab-treated M0 口Wnt5a+lgG-treated MO

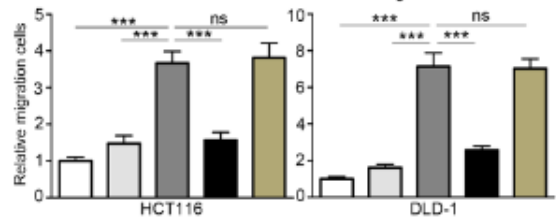

G $\square$ Ctrl $\square$ MO $\square$ Wnt5a-treated MO - Wnt5a+lL-10 Ab-treated M0 口 Wnt5a+lgG-treated M0

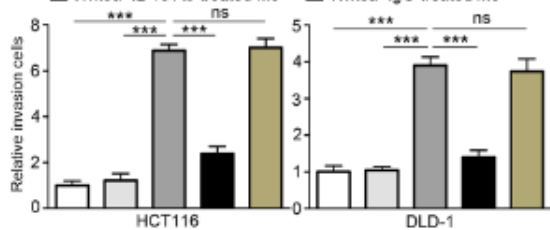

\section{Figure 5}

Wnt5a indirectly promotes tumor proliferation, migration and invasion through M2 macrophages. a Flow chart of CRC cells co-cultured with M0 macrophages treated with Wnt5a or Wnt5a+IL-10 antibody or Wnt5a+lgG. b, c Representative photographs and quantifications of clone formation assay in HCT116 and DLD-1 cells co-cultured with conditioned MO macrophages. Bar $=0.5 \mathrm{~cm}$. d, e Transwell migration assay of HCT116 and DLD1 cells co-cultured with conditioned M0 macrophages. Bar $=200 \mu \mathrm{m}$. f, g Transwell invasion assay of HCT116 and DLD-1 cells co-cultured with conditioned M0 macrophages. Bar $=200 \mu \mathrm{m}$. All experiments were performed in triplicate. Statistical analysis was conducted using one-way ANOVA. Error bars, SEM. ns, not significant. *** $\mathrm{P}<0.001$ 

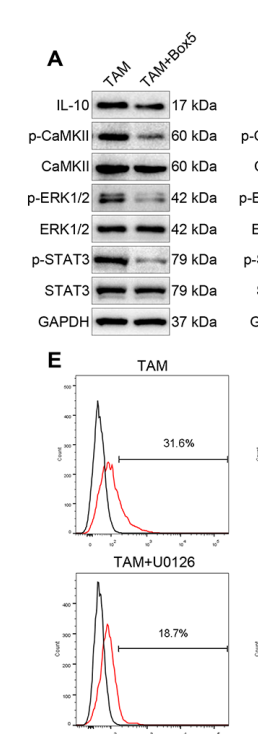

F

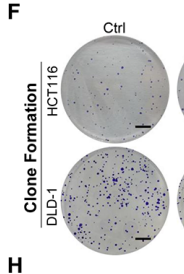

H

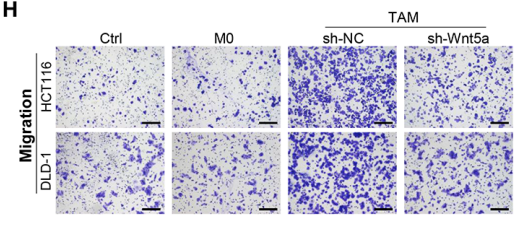

$\mathbf{J}$
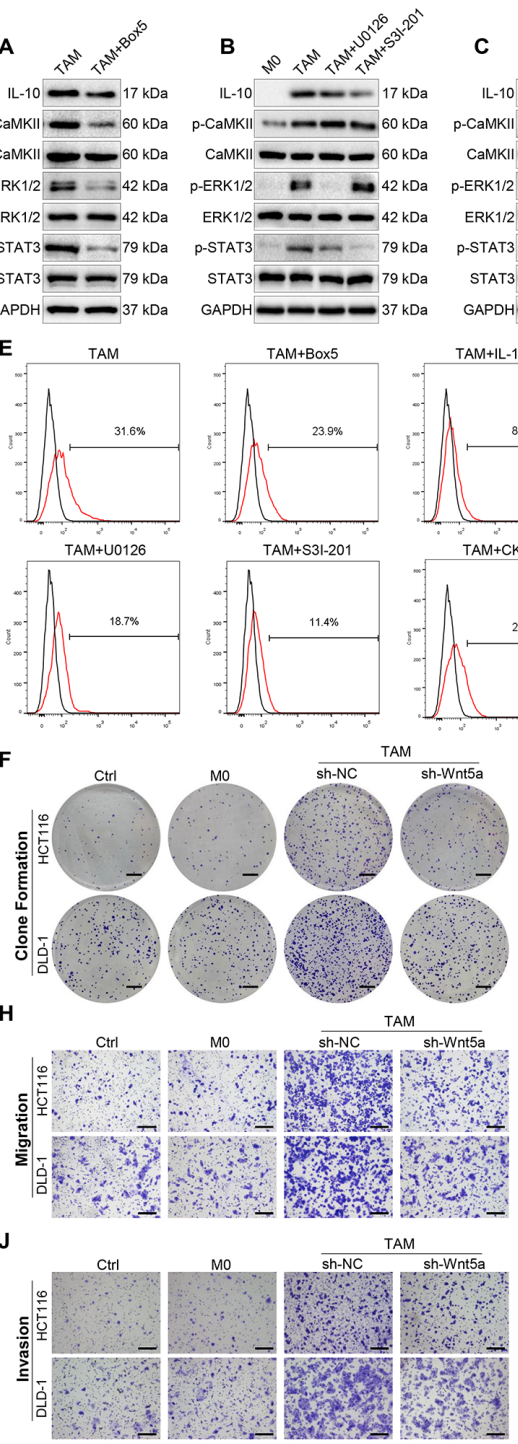

TAM+S31-201

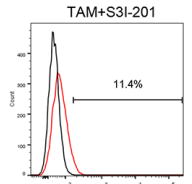

TAM

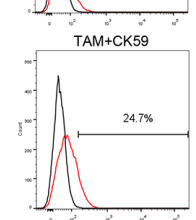

TAM+1L-10Ab
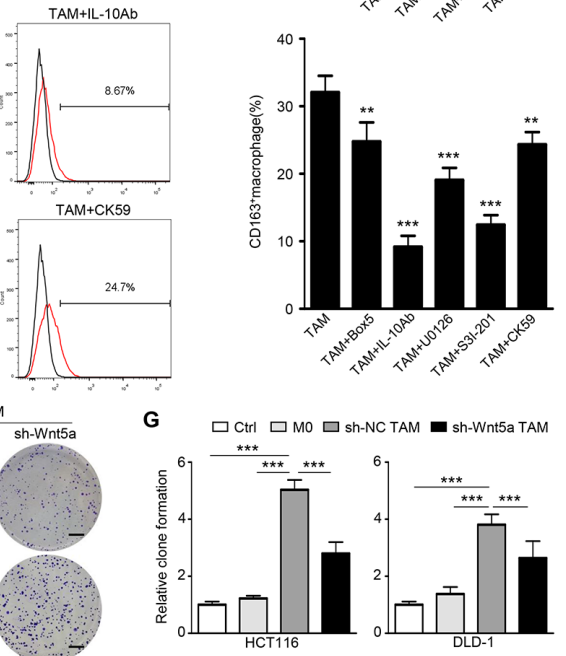

I $\square$ Ctrl $\square$ MO $\square$ sh-NC TAM - sh-Wnt5a TAM

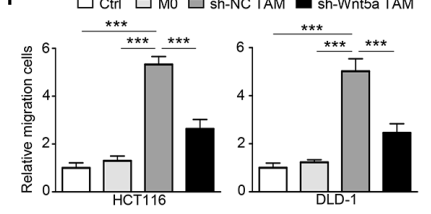

K $\square$ Ctrl $\square$ MO $\square$ sh-NC TAM $\mathbf{a}$ sh-Wnt5a TAM

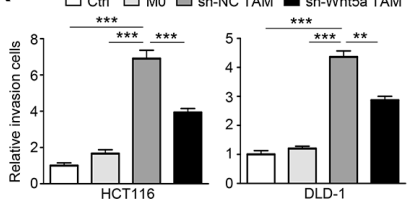

Figure 6

Wnt5a is crucial for the pro-tumor functions of TAMs. a Western blot analysis of TAMs with or without Box5. b Western blot analysis of TAMs with or without U0126 or S3I-201. c Western blot analysis of TAMs with or without CK59. d ELISA detected the secretion of IL-10 in TAMs treated with inhibitors. Error bars, SEM. e Flow cytometry analysis of TAMs with or without inhibitors. Error bars, SEM. f, g Representative photographs and quantification of clone formation assay in HCT116 and DLD-1 cells co-cultured with M0 macrophages and TAMs transfected with sh-Wnt5a or shNC. Bar $=0.5 \mathrm{~cm}$. Error bars, SEM. h, i Transwell migration assay of HCT116 and DLD-1 cells co-cultured with conditioned macrophages. Error bars, SEM. Bar $=200 \mu \mathrm{m}$. j, $\mathrm{k}$ Transwell invasion assay of HCT116 and DLD-1 cells co-cultured with conditioned macrophages. Error bars, SEM. Bar $=$ $200 \mu \mathrm{m}$. All experiments were performed in triplicate. Statistical analysis was conducted using one-way ANOVA. ${ }^{*} P<0.05 .{ }^{* \star P}<0.01$. ${ }^{\star * *} \mathrm{P}<0.001$ 


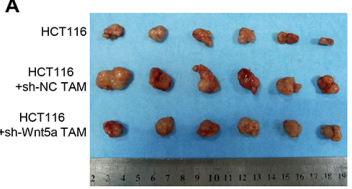

C
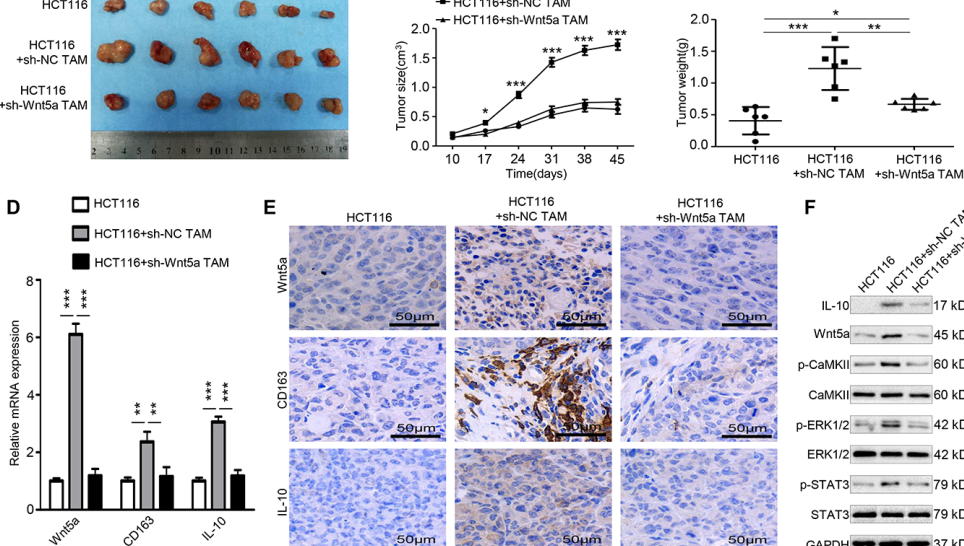

E HCT116
+ +sh-NC TAM
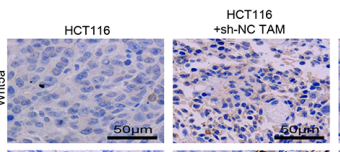

HCT116
+5h-Wnt5a TAM

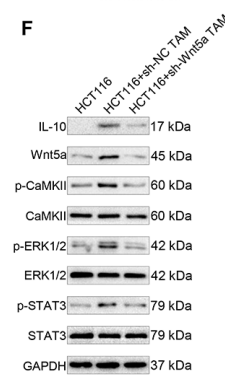

G
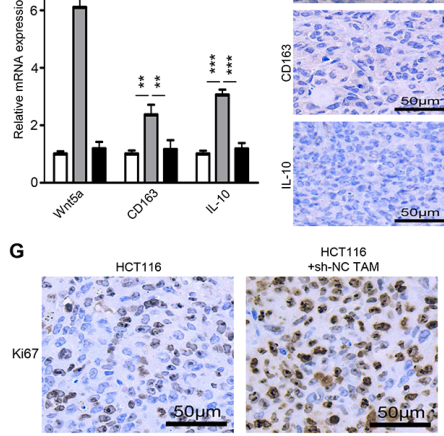

1. 3.
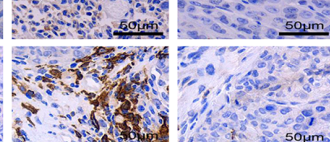

$17 \mathrm{kDa}$

Wnita - $-45 \mathrm{kDa}$
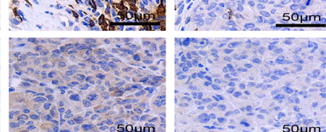

HCT116
TSh-NC TAM
The

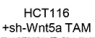
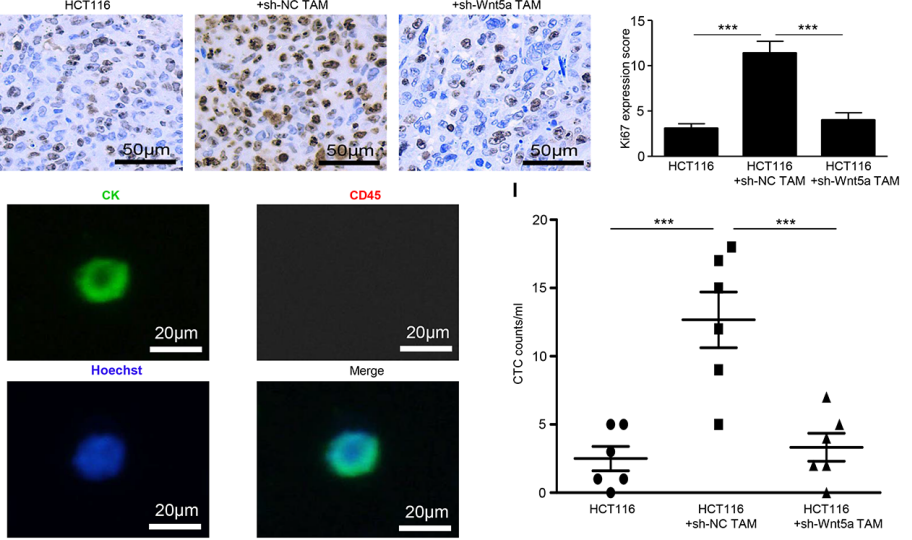

J HCT116+Sh-NC TAM
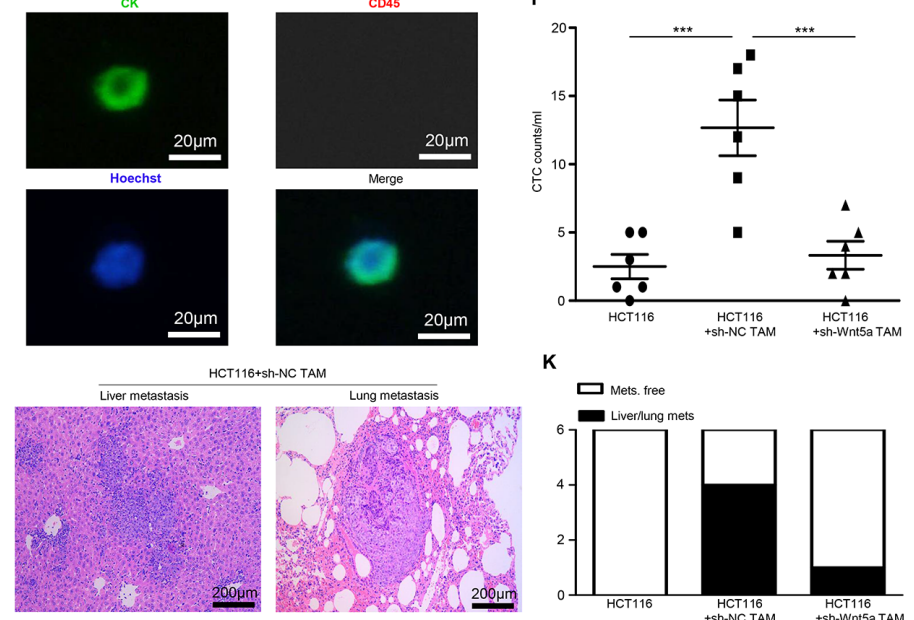

K

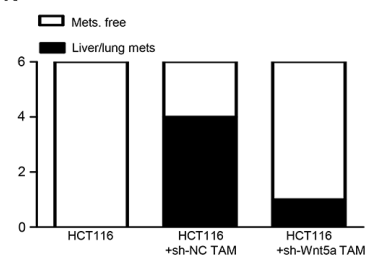

Figure 7

Silencing Wnt5a suppresses M2-like TAM-mediated tumor growth and metastasis in vivo. a-c The morphological characteristics of tumor xenograft, tumor size and tumor weight in the HCT116 alone, HCT116+sh-NC TAM and HCT116+sh-Wnt5a TAM groups. Error bars, SEM. d Relative mRNA expression of Wnt5a, CD163 and IL-10 of tumors in different groups. Error bars, SEM. e IHC analyzed the expression of Wnt5a, CD163 and IL-10 protein of tumors in different groups. Bar $=50 \mu \mathrm{m}$. $\mathrm{f}$ Western blot analysis of Wnt5a, IL-10, p-CaMKII, p-ERK1/2, and p-STAT3 expression in different groups. g IHC analyzed Ki67 expression of tumors in different groups. Bar $=50 \mu \mathrm{m}$. Error bars, SEM. $\mathrm{h}$ Representative CTC images of immunofluorescence. Bar $=20 \mu \mathrm{m}$. i Quantification of CTCs captured from the blood of mice. Error bars, SEM. j Representative H\&E stained sections of metastatic nodules in liver and lung tissue from mice in the HCT116+ sh-NC TAM group were shown. Bar $=200 \mu \mathrm{m}$. $\mathrm{k}$ Quantification of mice with metastasis lesions in different groups. Statistical analysis was conducted using one-way ANOVA. ${ }^{*} P<0.05 .{ }^{* *} P<0.01$. ${ }^{* * * P<0.001}$

\section{Supplementary Files}

This is a list of supplementary files associated with this preprint. Click to download.

- Supplementarymaterials.docx 